\title{
Frontal lobe neurology and the creative mind
}

\section{Leonardo C. de Souza ${ }^{1}$, Henrique C. Guimarães ${ }^{1}$, Antônio L. Teixeira ${ }^{1}$, Paulo Caramelli' Richard Levy ${ }^{2,3,4,5,6}$, Bruno Dubois ${ }^{2,3,4,5,7}$ and Emmanuelle Volle (2,3,5* $^{2,4}$}

\author{
${ }^{1}$ Neuropsychiatric Branch, Neurology Division, University Hospital, Universidade Federal de Minas Gerais, Belo Horizonte, Brazil \\ 2 Inserm, U 1127, ICM Frontlab, Paris, France \\ ${ }^{3}$ CNRS, UMR 7225, ICM Frontlab, Paris, France \\ ${ }^{4}$ Sorbonne Universités, UPMC Univ Paris 06, UMR S 1127, Paris, France \\ ${ }^{5}$ Institut du Cerveau et de la Moelle épinière, ICM Frontlab, Paris, France \\ ${ }^{6}$ AP-HP, Hôpital Saint-Antoine, Service de Neurologie, Paris, France \\ ${ }^{7}$ AP-HP, Hôpital de la Salpétrière, Neurology Department, Institut de la Mémoire et de la Maladie d'Alzheimer, Paris, France
}

\section{Edited by:}

Anna Abraham, Kuwait University,

Kuwait

Reviewed by:

Hugues Duffau, Montpellier

University Medical Center and

INSERM U1051, France

Dahlia Zaidel, University of

California, USA

*Correspondence:

Emmanuelle Volle, ICM Research Center, Hôpital Pitié Salpêtrière, 47, bd de l'hôpital, 75013 Paris, France e-mail:emmavolle@gmail.com
Concepts from cognitive neuroscience strongly suggest that the prefrontal cortex (PFC) plays a crucial role in the cognitive functions necessary for creative thinking. Functional imaging studies have repeatedly demonstrated the involvement of PFC in creativity tasks. Patient studies have demonstrated that frontal damage due to focal lesions or neurodegenerative diseases are associated with impairments in various creativity tasks. However, against all odds, a series of clinical observations has reported the facilitation of artistic production in patients with neurodegenerative diseases affecting PFC, such as frontotemporal dementia (FTD). An exacerbation of creativity in frontal diseases would challenge neuroimaging findings in controls and patients, as well as the theoretical role of prefrontal functions in creativity processes. To explore this paradox, we reported the history of a FTD patient who exhibited the emergence of visual artistic productions during the course of the disease. The patient produced a large amount of drawings, which have been evaluated by a group of professional artists who were blind to the diagnosis. We also reviewed the published clinical cases reporting a change in the artistic abilities in patients with neurological diseases. We attempted to reconcile these clinical observations to previous experimental findings by addressing several questions raised by our review. For instance, to what extent can the cognitive, conative, and affective changes following frontal damage explain changes in artistic abilities? Does artistic exacerbation truly reflect increased creative capacities? These considerations could help to clarify the place of creativity-as it has been defined and explored by cognitive neuroscience-in artistic creation and may provide leads for future lesion studies.

Keywords: creativity, prefrontal cortex, frontotemporal dementia, artistic, divergent thinking
Beyond its cultural, aesthetic or artistic aspects, creativity can be defined from a neuroscientific perspective as "the ability to produce a work that is both original (new, unusual, novel, unexpected) and valuable (useful, good, adaptive, appropriate)" (Sternberg and Lubart, 1999; Dietrich, 2004). Creative thinking usually involves the ability to break with conventional wellestablished ideas and to develop alternative behaviors in new and unexpected situations. In this sense, creativity may be considered to be a particular form of adaptation or problem solving (Runco, 2004; Sternberg, 2006). In this theoretical view, creativity relies on fundamental cognitive processes such as working memory, attention, planning, cognitive flexibility, mentalizing, and abstract thinking (Carlsson et al., 2000; Dietrich, 2004; Bogousslavsky, 2005; Changeux, 2005). These functions depend largely on the integrity of the prefrontal cortex (PFC), a brain region that is essential for behavioral adaptation and highly integrated mental functions. Functional neuroimaging data in healthy subjects also show that the PFC plays an important role in the cognitive processes involved in creativity (Gonen-Yaacovi et al., 2013).
Therefore, both cognitive theories and neuroimaging data suggest that the integrity of the PFC is essential for creative thinking, and that neurological diseases that damage PFC regions (or their connections) would affect cognitive creativity processes. Some experimental studies have indeed demonstrated the impairment of creativity after prefrontal damage (Rankin et al., 2007; de Souza et al., 2010; Shamay-Tsoory et al., 2011; Abraham et al., 2012).

However, in contrast with these theories and experiments, a series of clinical observations reports the facilitation of artistic abilities in some patients with neurodegenerative disease affecting the frontal lobes, raising the question of a possible increased creativity following frontal damage (Palmiero et al., 2012; Schott, 2012; Gretton and ffytche, 2014). An exacerbation of creativity in neurological diseases affecting the frontal lobes would question the role of the PFC in creativity.

Herein, we propose that cognitive aspects of creativity depend on the integrity of PFC subregions and we hypothesize that some of these contradictory data may be reconciled by considering the repercussion of frontal symptoms into the patients' production, 
by taking into account affective and conative aspects of creativity, and by comparing the artistic and neuroscientific perspectives of creativity. This discussion will be illustrated using a clinical case of artistic production during the course of the behavioral variant frontotemporal dementia (bvFTD).

\section{PREFRONTAL FUNCTIONS AND CREATIVITY}

The PFC is highly developed in humans and plays a crucial role in elaborating and controlling voluntary and goal-directed behaviors, expanding behavior far beyond the sole repertoire of automatic and reflexive actions. The PFC enables adaptive behavior according to one's own objectives and to the context while taking into account past experiences and needs (Goldman-Rakic, 1995; Shallice and Burgess, 1996; Fuster et al., 2000; Miller and Cohen, 2001; Levy and Volle, 2009; Volle et al., 2013). This central role in adaptive behavior is supported by intense connections between the PFC and other brain regions (Dubois et al., 1995; Mesulam, 1998). The strong connective properties of this region suggest that the PFC is involved in integrating or combining different types of information according to the task goal. The PFC is connected with the sensory systems involved in perception, enabling access to information about the current environment. The PFC receives information about past events and knowledge though connections to long-term memory circuits. The PFC is also part of the limbic system and receives information on the individual needs, emotions, and motivations (Schoenbaum et al., 2009; Fellows, 2013) to guide decisions. The PFC interacts with motor systems that program, perform and monitor the plan of actions (Catani and Thiebaut de Schotten, 2012; Yeterian et al., 2012; Cole et al., 2013; Rojkova et al., under revision). Thus, the PFC can be considered to be a convergence hub that enables the integration of different types of information and the formation of mental representations of both the external and inner worlds (Ramnani and Owen, 2004; Reynolds et al., 2006; Nee et al., 2013) that can guide more sophisticated patterns of behavior.

Furthermore, the connections between the PFC and other brain regions are usually reciprocal, enabling the PFC to exert control over other brain systems, in addition to receiving information. For instance, control signals over the action system may inhibit actions that would not be suitable in a given context, and control over perceptual systems enables the selection of relevant information in the environment (Picton et al., 2007; Levy and Wagner, 2011; Volle et al., 2012). The supervisory role of the PFC also allows the selection and the voluntary retrieval of information in memory (Martin and Cheng, 2006; Thompson-Schill and Botvinick, 2006; Badre and Wagner, 2007; Strenziok et al., 2013). Several recent models describe a hierarchical postero-anterior organization of the control functions that are exerted by PFC in which an increased control requirement for behavioral adaptation recruits more anterior PFC subregions (Koechlin et al., 2003; Koechlin and Hyafil, 2007; Azuar et al., 2014). Other models also describe a posteroanterior PFC gradient in the abstraction degree of the mental representations that can be formed; more anterior regions support more abstract thinking (Christoff et al., 2001, 2009; Badre and Wagner, 2007; Volle et al., 2010).

Overall, the PFC enables the formation and control of mental representations according to an internal goal by selecting information from the environment or from memory, by forming or selecting rules, and by resisting spontaneous prepotent responses (Levy and Volle, 2009). These prefrontal properties are assumed to support creativity as well as complex human abilities such as planning, reasoning, problem solving, abstract thinking (Carlsson et al., 2000; Godefroy, 2003; Dietrich, 2004; Bogousslavsky, 2005; Changeux, 2005; Burgess et al., 2009; Levy and Volle, 2009). In other words, our knowledge of PFC structure and functions supports the assumption that the PFC is essential for cognitive processes that underlie creative thinking. Experimental studies using creativity tasks in healthy participants and in patients confirm this hypothesis.

\section{EXPERIMENTAL STUDIES ON THE NEURAL CORRELATES OF CREATIVITY FUNCTIONAL NEUROIMAGING APPROACH: A ROLE FOR THE FPC IN CREATIVITY}

Functional imaging studies have attempted to explore the cerebral bases of creativity processes using various experimental tasks (see Arden et al., 2010; Dietrich and Kanso, 2010; Jung, 2013 for reviews). Some studies relied on ecological tasks attempting to imitate creativity in real life, but most of them employed tasks drawn from theoretical cognitive models. Studies with a more ecological approach used tasks such as story writing (Bechtereva et al., 2004; Howard-Jones et al., 2005; Shah et al., 2013), object design (Kowatari et al., 2009; Ellamil et al., 2012), or music improvisation (Bengtsson et al., 2007; Berkowitz and Ansari, 2008; Limb and Braun, 2008; de Manzano and Ullen, 2012).

Among the studies based on theory-based creativity tasks, the most frequent framework used to examine the brain correlates of creativity was the divergent thinking approach (Runco and Acar, 2012). Divergent thinking tests typically require generating the maximal number of new or unusual responses. One of the classical divergent thinking tasks is the Alternate Uses task, which assesses the ability to produce many alternative uses of a common object such as a brick.

Another approach, which was proposed by Mednick (Mednick, 1962; Mednick et al., 1964), considers that creativity results from "the forming of associative elements into new combinations, which either meet specified requirements or are in some way useful. The more mutually remote the elements of the new combination, the more creative the process or solution." One experimental task to test this hypothesis is to present three unrelated words without obvious connections between them (e.g., stain, glass, and red), and to ask the subject to find a fourth word that is related to each of these words (e.g., wine) (Jung-Beeman et al., 2004; Kounios et al., 2006). This task has been mainly used to investigate the phenomenon of "insight" or "Aha!" or "Eureka" (Kounios et al., 2006). "Aha” describes a subjective experience that occurs when solving a problem for which the solution suddenly comes to mind without effort or difficulty and is associated with a feeling of pleasure and confidence (Luo et al., 2004; Aziz-Zadeh et al., 2009; Qiu et al., 2010; Tian et al., 2011). This "Aha" experience is the cornerstone of another approach in creativity studies, that of problem solving with insight. Problems that raise an insight phenomenon include statements with strong implicit constraints that guide the 
search for a solution in the incorrect direction. The solution to these problems requires breaking these constraints and implicit associations and opening the search space to more possibilities. According to the classical model from Wallas (Kozbelt, 2011), this element is part of a creative process that follows four stages. Insight follows a preparation and an incubation phases and is followed by a verification phase. For many authors, the creative process is not this linear but instead alternates between phases of idea generation, evaluation, and the selection of ideas (Changeux, 2005; Simonton, 2010; Ward and Kolomyts, 2010; Ellamil et al., 2012).

A recent coordinate-based meta-analysis (Gonen-Yaacovi et al., 2013) using GingerALE free software (Eickhoff et al., 2012; http:www.brainmap.org/ale/) reviewed the published data regarding the investigation of the neural basis of creative thinking in functional neuroimaging studies. This study included 34 articles reporting 44 different experiments that employed the different creative paradigms aforementioned, i.e., divergent thinking tasks (Seger et al., 2000; Howard-Jones et al., 2005; Asari et al., 2008; Fink et al., 2009, 2010; Chrysikou and Thompson-Schill, 2011; Abraham et al., 2012; Ellamil et al., 2012; Kröger et al., 2012; Rutter et al., 2012) combination tasks and problem solving (Jung-Beeman et al., 2004; Luo et al., 2004; Geake and Hansen, 2005; Vartanian and Goel, 2005; Kounios et al., 2006; Mashal et al., 2007; Siebörger et al., 2007; Aziz-Zadeh et al., 2009; Qiu et al., 2010; Tian et al., 2011; Aziz-Zadeh et al., 2012; Cardillo et al., 2012; Green et al., 2012; Huang et al., 2013), as well as ecological tasks attempting to capture real life creativity instead of hypothesized cognitive processes (Bechtereva et al., 2004; Howard-Jones et al., 2005; Bengtsson et al., 2007; Berkowitz and Ansari, 2008; Limb and Braun, 2008; Kowatari et al., 2009; Ellamil et al., 2012; de Manzano and Ullen, 2012; Shah et al., 2013).

Despite the diversity of tasks used in these studies, the results showed a common set of brain regions as the neural basis of creative thinking, including multiple areas within the PFC and regions involved in semantic memory (the temporo-parietal region and posterior temporal and antero-lateral temporal cortex).

Additionally, this meta-analysis showed that distinct prefrontal subregions support distinct cognitive creativity processing. More specifically, tasks based on divergent thinking (to imagine alternative uses of objects or new designs) and those requiring the combination of information (to compose a sentence with unrelated words or to combine different figures to produce a new one, e.g.) were associated with both common and distinct prefrontal areas. Caudal lateral PFC was involved in both task categories, while more anterior PFC areas appear to be more task-oriented. For instance, within the frontal pole, the lateral part was more related to combination tasks, while its medial portion was engaged in divergent thinking tasks.

Together, these findings underlie the importance of PFC in creativity and suggest that different processes involved in creative thinking rely on distinct subregions within the PFC, in particular along the posterior-anterior axis and the medial-lateral axis. If PFC subregions are involved in creativity tasks, as suggested by functional imaging, one expects that damage to these areas would provoke impairment in the same tasks.

\section{EXPERIMENTAL PATIENT STUDIES: DECREASED CREATIVITY AFTER PREFRONTAL DAMAGE}

Whether PFC regions are critical to creativity has been explored in very few patient studies. Creative thinking has been studied in patients with focal brain lesions (Shamay-Tsoory et al., 2011; Abraham et al., 2012) and in one of the most frequent causes of frontal damage: frontotemporal dementia (FTD) (Rankin et al., 2007; de Souza et al., 2010). FTD is a neurodegenerative disease and the second most common cause of dementia in patients under 65 years of age. FTD encompasses three different clinical syndromes: the behavioral variant (bvFTD) and the language variants, i.e., progressive non-fluent aphasia and semantic dementia (SD).

de Souza et al. (2010) investigated creativity in patients with bvFTD, using a standardized test of divergent thinking, the Torrance Test of Creative Thinking (TTCT; Torrance, 2004). The TTCT includes both verbal and figurative tasks. TTCT establishes objective criteria to measure creative production, by scoring three main aspects: (1) the fluency, i.e., the total number of responses, (2) the flexibility, i.e., the number of different categories to which the responses belong, and (3) originality, which is the number of new responses, here considered as responses that are statistically infrequent. Fluency and flexibility are usually defined as executive functions and are classically assessed in neuropsychological testing. The results from de Souza and colleagues showed that bvFTD patients performed worse than controls (a normal and a pathological control group) in all dimensions of the TTCT (fluency, flexibility, and originality) for both figurative and verbal tasks. bvFTD patients had also impaired performance in frontal functions such as flexibility, inhibition, abstraction and planning. These findings are consistent with previous data demonstrating that bvFTD patients have impairments in the production of new ideas either in an ecological task of artistic drawing or on the TTCT (Rankin et al., 2007). This study also showed that behavioral disorders such as perseverations and behavioral disinhibition (often sexual) could partly account for the "originality" of frontal patients in their responses in TTCT. In other words, some of the production features may be considered to be manifestations of the behavioral disorders that characterize bvFTD; these were not observed in the control subjects.

In this study, brain correlates of creative abilities were also explored in bvFTD patients, and perfusion in prefrontal regions measured using SPECT correlated with creativity performance at the TTCT (de Souza et al., 2010). More interestingly, there was a clear concordance among the regions reported in this study and those observed in functional neuroimaging studies in healthy subjects (Gonen-Yaacovi et al., 2013), in particular in the left inferior frontal gyrus [BA 47], the left posterior inferior and middle temporal gyri [BA 37], the left inferior parietal lobule [BA39/40], and the left precuneus [BA 23].

Focal prefrontal lesions also impact creative thinking, as demonstrated by two recent lesion studies that examined the consequences of focal brain damage (such as stroke) on creative performance (Shamay-Tsoory et al., 2011; Abraham et al., 2012). Shamay-Tsoory et al. (2011) compared patients' performance on the TTCT according to distinct lesion locations: frontal pole, posterior part of the PFC, or outside the PFC. The results showed that 
damage to the frontal pole was specifically associated with a deficit at the TTCT. More especially, the originality criterion was the most compromised, and patients with damage to the frontal pole were less original in their response than other patients. Abraham et al. (2012) used several creativity tests in patients with various lesion locations and showed that patients with lateral frontal damage were impaired in both fluency and originality aspects of divergent thinking tasks.

Taken together, these data supports the critical role of PFC in creative thinking. From a cognitive perspective, cerebral findings from patient studies agree with functional neuroimaging results (Carlsson et al., 2000; Seger et al., 2000; Bechtereva et al., 2004; Jung-Beeman et al., 2004; Goel and Vartanian, 2005; HowardJones et al., 2005; Asari et al., 2008; Aziz-Zadeh et al., 2009; Fink et al., 2009, 2010; Kowatari et al., 2009). These findings are also consistent with studies that used SPECT (Chavez-Eakle et al., 2007), voxel-based morphometry (Jung et al., 2010b; Takeuchi et al., 2010a; Gansler et al., 2011), and diffusion tensor imaging (Jung et al., 2010a; Takeuchi et al., 2010b).

However, against all odds, a series of medical observations have reported the facilitation of artistic abilities in patients with damage to the frontal lobes (Palmiero et al., 2012; Schott, 2012).

\section{CLINICAL OBSERVATIONS OF CREATIVITY IN NEUROLOGICAL PATIENTS}

The description of patients developing artistic abilities raises the question of enhanced creativity following frontal damage, which would challenge the neuroimaging findings in controls and patients and the theoretical role of prefrontal functions in creativity processing. To better understand the relationships between frontal damage, frontal functions, artistic ability, and creativity, we performed a mini-review of published articles reporting changes in artistic production by neurological patients.

\section{A MINI-REVIEW OF MEDICAL REPORTS ON CREATIVITY}

We actively searched the PubMed database for previous medical reports of changes in artistic skills in neurological patients. Unlike experimental studies on creativity that were usually based on various experimental tasks using objective measures and more instructed tasks, these clinical reports were based on a subjective evaluation of spontaneous patients' productions in the artistic domain. We used the following key-words terms: "dementia, frontotemporal+dementia, Alzheimer's+disease, semantic+dementia, or stroke" AND "creativity, artistry, or artist." We looked for articles published until March 2014. We also included articles cited in previous reviews on creativity in patients (Palmiero et al., 2012; Schott, 2012; Gretton and ffytche, 2014). We did not include Parkinson disease, as artistic facilitation in this condition may most likely relate to the dopa medication rather than to the brain damage itself (Lhommee et al., 2014). The papers found throughout this research were evaluated for relevance and duplicate cases were excluded.

We found 35 relevant papers reporting the degradation, emergence, preservation or improvement of creative expression in 53 patients after the onset of different neurological diseases (see Table 1): 19 patients with temporal variant FTD (semantic dementia), 10 patients with behavioral variant FTD, eight patients with Alzheimer's disease, four patients with primary progressive non-fluent aphasia, and 12 patients with various neurological diseases (Espinel, 1996; Miller et al., 1998, 2000; Crutch et al., 2001; Thomas-Anterion et al., 2002, 2010; Kleiner-Fisman et al., 2003; Mell et al., 2003; Mendez and Perryman, 2003; Annoni et al., 2005; Fornazzari, 2005; Lythgoe et al., 2005; Serrano et al., 2005; Chatterjee et al., 2006; Drago et al., 2006a,b; Budrys et al., 2007; Finney and Heilman, 2007; Midorikawa et al., 2008; Seeley et al., 2008; Liu et al., 2009; Thomas-Anterion, 2009; Chakravarty, 2011; Chatterjee et al., 2011; van Buren et al., 2013; Galarza et al., 2014; Takahata et al., 2014). All reported patients with temporal FTD $(n=19)$ presented the emergence $(n=11)$, increase $(n=2)$, or preservation $(n=6)$ of creative production but no degradation of artistic abilities (Miller et al., 1996, 1998; Edwards-Lee et al., 1997; Drago et al., 2006b; Wu et al., 2013). Most case reports on behavioral variant FTD $(n=10)$ noted the emergence $(n=$ $4)$, increase $(n=4)$, or preservation $(n=1)$ of artistic abilities (Miller et al., 1998; Thomas-Anterion et al., 2002; Mendez and Perryman, 2003; Serrano et al., 2005; Liu et al., 2009; ThomasAnterion, 2009). The effects of Alzheimer's disease on artistic production were more heterogeneous, with observations of both increase (Fornazzari, 2005; Chakravarty, 2011) and degradation (Cummings and Zarit, 1987; Crutch et al., 2001; Serrano et al., 2005; van Buren et al., 2013). Other neurological degenerative diseases or strokes of various locations were associated with heterogeneous profiles (Annoni et al., 2005; Lythgoe et al., 2005; Thomas-Anterion et al., 2010; Takahata et al., 2014). The cognitive, behavioral, and artistic changes reported in the reviewed studies are synthetized in Table 2 .

This non-systematic review highlights that some FTD patients develop enhanced artistic abilities and suggests that the relations between FTD, frontal functions, artistic abilities and creativity are unclear, as discussed below. We first would like to illustrate the paradoxical relationship between frontal symptoms and creativity by reporting the clinical observation of a patient who developed artistic abilities during the course of bvFTD. This is a new clinical case (unpublished original data) that will be discussed in conjunction with the other reviewed findings.

\section{CLINICAL VIGNETTE}

Mrs. YCFZ (case number 963564), a retired dentist secretary aged 83 years, was evaluated in October 2010 in the Cognitive and Behavioral Neurology Unit of the Clinics Hospital from the Federal University of Minas Gerais (Belo Horizonte, Brazil). She was referred to the unit for the evaluation of behavioral and cognitive symptoms that had been evolving for approximately 2 years. Her preceding medical history was unremarkable, except for systemic hypertension, which was well controlled.

The family reported that the patient demonstrated striking behavioral changes. She was progressively uninterested in previously appreciated household chores, and she narrowed her usual cooking repertoire, abandoning the preparation of traditional dishes from her native country, El Salvador. Increased appetite manifested as a troublesome binge eating cookies. Additionally, the patient became progressively less concerned with personal grooming. The patient developed a new stereotyped and fixed routine. For example, she started to eat one banana every day at 
Table 1 | Synthesis of published articles reporting changes in artistic creativity in neurological patients.

\begin{tabular}{|c|c|c|c|c|c|c|}
\hline Author and year & Diagnosis & Neuroimaging data & $\begin{array}{l}\text { Change in } \\
\text { abilities }\end{array}$ & $\begin{array}{l}\text { Previous interest in } \\
\text { Art? }\end{array}$ & $\begin{array}{l}\text { Art } \\
\text { domain }\end{array}$ & Neuropsychological data \\
\hline $\begin{array}{l}\text { Miller et al., 1998, } \\
2000 \\
\text { (Pt 3) }\end{array}$ & Frontal FTD & $\begin{array}{l}\text { SPECT: bifrontal and } \\
\text { temporal hypoperfusion } \\
\text { (right }>\text { left) }\end{array}$ & $E$ & $\begin{array}{l}\text { Occasionally } \\
\text { produced novels (not } \\
\text { a professional) }\end{array}$ & Photo & $\begin{array}{l}\text { MMSE }=26 / 30 \\
\text { Preserved language and } \\
\text { constructions } \\
\text { Impaired executive tests (WCST, } \\
\text { Stroop, TMT) } \\
\text { Behavioral disinhibition and } \\
\text { compulsions }\end{array}$ \\
\hline
\end{tabular}

\begin{tabular}{lll}
\hline Thomas-Anterion & Frontal FTD & CT scan: frontotemporal \\
et al., 2002 and & & atrophy \\
Thomas-Anterion, & SPECT: frontal \\
2009 & hypoperfusion
\end{tabular}

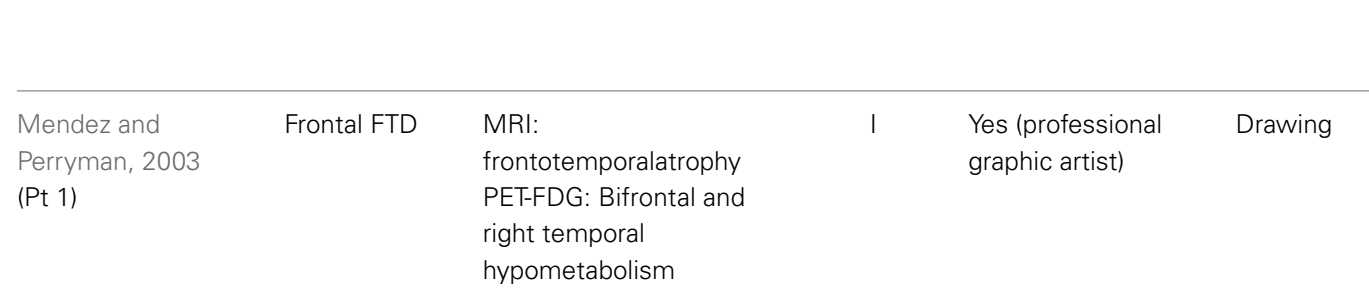

Language and memory
impairment
Impaired executive tests

Emotional difficulties

Apathy

Stereotypies

MMSE $=22 / 30$

Preserved language, face processing and visuospatial tests Decreased verbal fluency Concrete interpretation of proverbs

Compulsions and hoarding

Poor insight

\section{Mendez and}

I Yes (professional photographer and graphic designer)
Perryman, 2003

Frontal FTD

MRI: normal

SPECT: Bifrontal and right temporal hypoperfusion

Drawing MMSE $=23 / 30$

Preserved visuospatial and face processing tests

Decreased verbal fluency, executive functions and memory Difficulties with proverbs Inappropriate social behaviors and compulsions

Loss of insight

\section{Mendez and}

Perryman, 2003

(Pt 3)
I Occasionally caricatures (not a professional)
Frontal FTD

MRI: frontotemporal

atrophy

SPECT: Frontal and right

anterior temporal

hypoperfusion
Drawing MMSE 20/30

Preserved visuospatial and face processing tests

Decreased verbal fluency and memory

Difficulties with similarities and

proverbs

Poor insight

Compulsions

Disinhibited behaviors, impulsivity

\begin{tabular}{|c|c|c|c|c|c|c|}
\hline $\begin{array}{l}\text { Mendez and } \\
\text { Perryman, } 2003 \\
\text { (Pt 4) }\end{array}$ & Frontal FTD & $\begin{array}{l}\text { MRI: frontotemporal } \\
\text { atrophy } \\
\text { SPECT: Bifrontal and } \\
\text { bitemporal hypoperfusion }\end{array}$ & $\mathrm{P}$ & $\begin{array}{l}\text { Yes (professional } \\
\text { artist) }\end{array}$ & $\begin{array}{l}\text { Not } \\
\text { specified }\end{array}$ & $\begin{array}{l}\text { MMSE } 23 / 30 \\
\text { Preserved visuospatial and face } \\
\text { processing tests } \\
\text { Decreased verbal fluency } \\
\text { Good proverb interpretation } \\
\text { Disinhibition of personal behavior } \\
\text { Compulsive behaviors }\end{array}$ \\
\hline $\begin{array}{l}\text { Serrano et al., } 2005 \\
\text { (Pt 3) }\end{array}$ & Frontal FTD & $\begin{array}{l}\text { MRI: normal } \\
\text { SPECT: Left } \\
\text { fronto-temporoparietal } \\
\text { hypoperfusion }\end{array}$ & 1 & Yes (painter) & Painting & $\begin{array}{l}\text { Impaired language skills } \\
\text { Impaired executive tests (TMT, } \\
\text { spans) } \\
\text { Preserved performance on } \\
\text { similarity test } \\
\text { Compulsive behaviors }\end{array}$ \\
\hline
\end{tabular}

(Continued) 
Table 1 | Continued

\begin{tabular}{|c|c|c|c|c|c|c|}
\hline Author and year & Diagnosis & Neuroimaging data & $\begin{array}{l}\text { Change in } \\
\text { abilities }\end{array}$ & $\begin{array}{l}\text { Previous interest in } \\
\text { Art? }\end{array}$ & $\begin{array}{l}\text { Art } \\
\text { domain }\end{array}$ & Neuropsychological data \\
\hline Liu et al., 2009 & Frontal FTD (a) & $\begin{array}{l}\text { MRI: atrophy in bilateral } \\
\text { anterior and left lateral } \\
\text { frontal regions. }\end{array}$ & $E$ & No & $\begin{array}{l}\text { Painting } \\
\text { Sculpture }\end{array}$ & $\begin{array}{l}\text { MMSE } 28 / 30 \\
\text { Preserved visuospatial skills } \\
\text { Impaired executive tests } \\
\text { Abstraction difficulties } \\
\text { Lack of emotion, empathy and } \\
\text { insight } \\
\text { Impaired verbal memory and } \\
\text { semantic } \\
\text { Antisocial and compulsive } \\
\text { behaviors } \\
\text { Paintings contain sexual } \\
\text { disinhibition } \\
\text { Obsessions about art and dots } \\
\text { and stripes }\end{array}$ \\
\hline $\begin{array}{l}\text { Thomas-Anterion, } \\
2009 \\
\text { (Pt 2) }\end{array}$ & Frontal FTD & No imaging data & $E$ & No & $\begin{array}{l}\text { Drawing } \\
\text { Poetry }\end{array}$ & $\begin{array}{l}\text { No neuropsychological data } \\
\text { Obsession about art }\end{array}$ \\
\hline Budrys et al., 2007 & Frontal FTD (b) & $\begin{array}{l}\text { MRI: bilateral } \\
\text { frontotemporal atrophy }\end{array}$ & $D$ & $\begin{array}{l}\text { Yes (professional } \\
\text { artist) }\end{array}$ & Painting & $\begin{array}{l}\text { MMSE } 25 / 30 \\
\text { Aphasia and amnesia } \\
\text { Difficulties on abstract reasoning } \\
\text { Verbal and writing perseverations }\end{array}$ \\
\hline $\begin{array}{l}\text { Edwards-Lee et al., } \\
1997 \\
\text { (Pt LTLV 1) } \\
\text { and Miller et al., } \\
2000\end{array}$ & Temporal FTD & $\begin{array}{l}\text { MRI: bitemporal atrophy, } \\
\text { SPECT: Bitemporal } \\
\text { hypoperfusion }\end{array}$ & $P$ & Yes (pianist) & Music & $\begin{array}{l}\text { MMSE }=1 / 30 \\
\text { Preserved attentional and } \\
\text { visuospatial skills } \\
\text { Impaired executive tests (Stroop, } \\
\text { TMT) } \\
\text { Compulsive behaviors }\end{array}$ \\
\hline $\begin{array}{l}\text { Edwards-Lee et al., } \\
1997 \\
\text { (Pt LTLV 3) } \\
\text { and Miller et al., } \\
2000\end{array}$ & Temporal FTD & $\begin{array}{l}\text { MRI: left temporal lobe } \\
\text { atrophy } \\
\text { SPECT: Left temporal } \\
\text { hypoperfusion }\end{array}$ & $P$ & Yes & $\begin{array}{l}\text { "Artistic } \\
\text { skills" }\end{array}$ & $\begin{array}{l}\text { MMSE 26/30 } \\
\text { Preserved visuospatial skills } \\
\text { Semantic anomia } \\
\text { Memory impairment }\end{array}$ \\
\hline $\begin{array}{l}\text { Edwards-Lee et al., } \\
1997 \\
\text { (Pt LTLV 5) } \\
\text { and Miller et al., } \\
2000\end{array}$ & Temporal FTD & $\begin{array}{l}\text { MRI: generalized atrophy } \\
\text { SPECT: Bitemporal } \\
\text { hypoperfusion }\end{array}$ & $E$ & No & Painting & $\begin{array}{l}\text { MMSE = 15/30 } \\
\text { Preserved visuospatial skills } \\
\text { Executive tests markedly } \\
\text { impaired (TMT, Stroop, verbal } \\
\text { fluency) } \\
\text { Anomic aphasia and impaired } \\
\text { memory }\end{array}$ \\
\hline $\begin{array}{l}\text { Miller et al., } 1998 \\
\text { and } 2000\end{array}$ & $\begin{array}{l}\text { Temporal FTD } \\
\text { (c) }\end{array}$ & $\begin{array}{l}\text { SPECT: bitemporal (Left } \\
>\text { right) and mild left } \\
\text { frontal hypoperfusion }\end{array}$ & $E$ & No & $\begin{array}{l}\text { Painting } \\
\text { drawing }\end{array}$ & $\begin{array}{l}\text { MMSE }=16 / 30 \\
\text { Preserved visuospatial skills } \\
\text { Letter fluency }=2 \\
\text { Perseverations on executive tests } \\
\text { Disinhibition and compulsive } \\
\text { behavior }\end{array}$ \\
\hline Miller et al., 1998 & Temporal FTD & No imaging data & $E$ & No & Painting & $\begin{array}{l}\text { No neuropsychological data } \\
\text { Disinhibition in language. }\end{array}$ \\
\hline $\begin{array}{l}\text { Miller et al., } 1998 \\
\text { and } 2000\end{array}$ & Temporal FTD & $\begin{array}{l}\text { MRI: bifrontal and left } \\
\text { temporal atrophy } \\
\text { SPECT: Left frontal and } \\
\text { bitemporal hypoperfusion }\end{array}$ & 1 & Yes & Sculpture & $\begin{array}{l}\text { MMSE }=9 / 30 \\
\text { Mild deficit in visuospatial tests } \\
\text { Decreased verbal fluency } \\
\text { Impaired memory and naming } \\
\text { Disinhibition and compulsive } \\
\text { behavior }\end{array}$ \\
\hline
\end{tabular}




\section{Table 1 | Continued}

\begin{tabular}{|c|c|c|c|c|c|c|}
\hline Author and year & Diagnosis & Neuroimaging data & $\begin{array}{l}\text { Change in } \\
\text { abilities }\end{array}$ & $\begin{array}{l}\text { Previous interest in } \\
\text { Art? }\end{array}$ & $\begin{array}{l}\text { Art } \\
\text { domain }\end{array}$ & Neuropsychological data \\
\hline $\begin{array}{l}\text { Miller et al., } 1998 \\
\text { and } 1998 \\
\text { (also in Miller et al., } \\
1996 \text { and } \\
\text { Edwards-Lee et al., } \\
1997 \text { Patient RTLV 4) }\end{array}$ & Temporal FTD & $\begin{array}{l}\text { MRI: bitemporal atrophy } \\
\text { SPECT: Bilateral temporal } \\
\text { hypoperfusion }\end{array}$ & $E$ & No & Painting & $\begin{array}{l}\text { MMSE }=15 / 30 \\
\text { Fluent verbal output, with } \\
\text { semantic anomia } \\
\text { Letter fluency }=2 \\
\text { Disinhibition and compulsive } \\
\text { behavior }\end{array}$ \\
\hline $\begin{array}{l}\text { Midorikawa et al., } \\
2008 \\
(\text { Pt 1) }\end{array}$ & Temporal FTD & $\begin{array}{l}\text { MRI: left temporal } \\
\text { atrophy }\end{array}$ & $E$ & No & Painting & $\begin{array}{l}\text { Language deficits (semantic } \\
\text { deficits) } \\
\text { Abnormal behaviors } \\
\text { (intrusiveness, repetitive actions) }\end{array}$ \\
\hline $\begin{array}{l}\text { Midorikawa et al., } \\
2008 \\
(\text { Pt 2) }\end{array}$ & Temporal FTD & $\begin{array}{l}\text { MRI: left temporal } \\
\text { atrophy }\end{array}$ & E & No & Painting & $\begin{array}{l}\text { Language deficits (semantic } \\
\text { deficits) }\end{array}$ \\
\hline $\begin{array}{l}\text { Miller et al., } 2000 \\
(\text { Pt 2) }\end{array}$ & Temporal FTD & $\begin{array}{l}\text { MRI: focal left temporal } \\
\text { atrophy } \\
\text { SPECT: bitemporal, left } \\
\text { greater than right, } \\
\text { hypoperfusion with } \\
\text { frontal sparing }\end{array}$ & $P$ & Yes (previous bridge) & Bridge & $\begin{array}{l}\text { MMSE }=25 / 30 \\
\text { Normal on Wisconsin Card Sort } \\
\text { Test } \\
\text { Normal visual reproduction } \\
\text { abilities } \\
\text { Intact social skills }\end{array}$ \\
\hline $\begin{array}{l}\text { Miller et al., } 2000 \\
(\text { Pt 3) }\end{array}$ & Temporal FTD & $\begin{array}{l}\text { SPECT: bitemporal } \\
\text { hypoperfusion with } \\
\text { frontal sparing }\end{array}$ & $\mathrm{P}$ & $\begin{array}{l}\text { Yes (previous } \\
\text { inventor) }\end{array}$ & Inventor & $\begin{array}{l}\text { MMSE }=22 / 30 \\
\text { Boston naming test: } 16 / 60 \\
\text { Apathy }\end{array}$ \\
\hline $\begin{array}{l}\text { Miller et al., } 2000 \\
\text { (Pt 5) }\end{array}$ & Temporal FTD & $\begin{array}{l}\text { SPECT: moderate left } \\
\text { temporal and mild left } \\
\text { frontal hypoperfusion }\end{array}$ & E & No & Music & $\begin{array}{l}\text { MMSE }=25 / 30 \\
\text { Decreased verbal output }\end{array}$ \\
\hline $\begin{array}{l}\text { Miller et al., } 2000 \\
\text { (Pt 6) }\end{array}$ & Temporal FTD & $\begin{array}{l}\text { Positron emission } \\
\text { tomography showed left } \\
\text { anterior hypometabolism }\end{array}$ & $P$ & Yes (music) & Music & $\begin{array}{l}\text { MMSE }=15 / 30 \\
\text { Fluent speech with } \\
\text { perseverations }\end{array}$ \\
\hline Drago et al., 2006a & Temporal FTD & $\begin{array}{l}\text { MRI: anterior bitemporal } \\
\text { atrophy }\end{array}$ & I & Yes (visual artist) & Painting & $\begin{array}{l}\text { Preserved visuospatial skills } \\
\text { Language deficits } \\
\text { Behavioral disorders (more } \\
\text { impulsive and belligerent) }\end{array}$ \\
\hline $\begin{array}{l}\text { Wu et al., } 2013 \\
\text { (Pt 1) }\end{array}$ & Temporal FTD & $\begin{array}{l}\text { MRI: bilateral (left greater } \\
\text { than right) anterior } \\
\text { temporal atrophy } \\
\text { extending to } \\
\text { hippocampal and } \\
\text { orbitofrontal regions }\end{array}$ & E & No & $\begin{array}{l}\text { Verbal } \\
\text { (poetry) }\end{array}$ & $\begin{array}{l}\text { MMSE } 26 / 30 \\
\text { Normal performance on visual } \\
\text { memory and visuospatial function } \\
\text { Impairment in verbal memory } \\
\text { Preserved executive function } \\
\text { Disinhibition }\end{array}$ \\
\hline
\end{tabular}


Table 1 | Continued

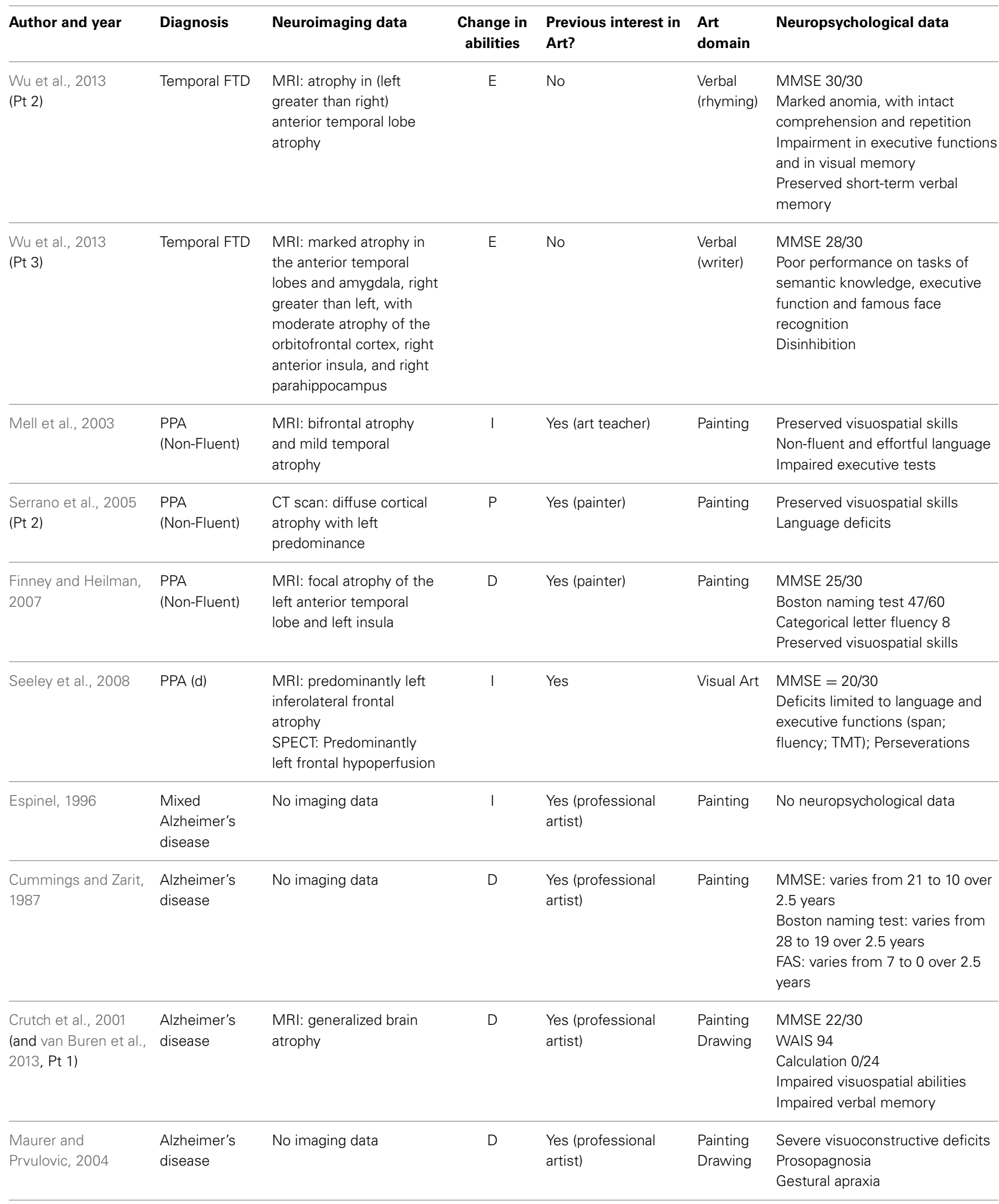

(Continued) 


\section{Table 1 | Continued}

\begin{tabular}{|c|c|c|c|c|c|c|}
\hline Author and year & Diagnosis & Neuroimaging data & $\begin{array}{l}\text { Change in } \\
\text { abilities }\end{array}$ & $\begin{array}{l}\text { Previous interest in } \\
\text { Art? }\end{array}$ & $\begin{array}{l}\text { Art } \\
\text { domain }\end{array}$ & Neuropsychological data \\
\hline Fornazzari, 2005 & $\begin{array}{l}\text { Alzheimer's } \\
\text { disease }\end{array}$ & $\begin{array}{l}\text { MRI: large arachnoid cyst } \\
\text { SPECT: Bilateral } \\
\text { temporo-parietal } \\
\text { hypoperfusion }\end{array}$ & $P$ & Yes (painter) & Painting & $\begin{array}{l}\text { MMSE 26/30 } \\
\text { Preserved visuospatial abilities } \\
\text { Deficits in episodic memory, } \\
\text { language, gestural praxis and } \\
\text { executive functions }\end{array}$ \\
\hline Chakravarty, 2011 & $\begin{array}{l}\text { Alzheimer's } \\
\text { disease }\end{array}$ & $\begin{array}{l}\text { CT scan: Diffuse cortical } \\
\text { atrophy }\end{array}$ & E & No & Painting & $\begin{array}{l}\text { MMSE }=16 / 30 \\
\mathrm{CDR}=3\end{array}$ \\
\hline $\begin{array}{l}\text { Kleiner-Fisman et al., } \\
2003\end{array}$ & $\begin{array}{l}\text { Corticobasal } \\
\text { degeneration }\end{array}$ & $\begin{array}{l}\text { MRI: right-predominant } \\
\text { atrophy } \\
\text { PET-FDG: marked } \\
\text { hypoperfusion on right } \\
\text { hemisphere and left } \\
\text { frontal region }\end{array}$ & $\mathrm{D}$ & $\begin{array}{l}\text { Yes (professional } \\
\text { illustrator) }\end{array}$ & $\begin{array}{l}\text { Graphic } \\
\text { Arts }\end{array}$ & $\begin{array}{l}\text { Severely impaired visuo-spatial } \\
\text { skills, spatial neglect } \\
\text { Deficits on attention, initiation, } \\
\text { memory and executive functions } \\
\text { Poor insight } \\
\text { Personality changes, irritability } \\
\text { Apathy }\end{array}$ \\
\hline Sahlas, 2003 & $\begin{array}{l}\text { Lewy Body } \\
\text { Dementia }\end{array}$ & No imaging data & $\mathrm{D}$ & $\begin{array}{l}\text { Yes (professional } \\
\text { artist) }\end{array}$ & $\begin{array}{l}\text { Painting } \\
\text { Writing }\end{array}$ & $\begin{array}{l}\text { No neuropsychological data } \\
\text { but reports of deterioration of } \\
\text { visuospatial functions }\end{array}$ \\
\hline $\begin{array}{l}\text { Annoni et al., } 2005 \\
\text { (Pt 2) }\end{array}$ & Stroke & $\begin{array}{l}\text { MRI: left paramedian } \\
\text { thalamus infarct }\end{array}$ & । & $\begin{array}{l}\text { Yes (professional } \\
\text { painter) }\end{array}$ & Painting & $\begin{array}{l}\text { MMSE }=28 / 30 \\
\text { Normal neuropsychological exam } \\
\text { Mild emotional dyscontrol } \\
\text { Moderate tendency to } \\
\text { perseveration in phonological and } \\
\text { figural fluency } \\
\text { No compulsive behaviors }\end{array}$ \\
\hline $\begin{array}{l}\text { Chatterjee et al., } \\
2011 \\
(\text { Pt 1) }\end{array}$ & Stroke & $\begin{array}{l}\text { No imaging data (left } \\
\text { hemisphere stroke) }\end{array}$ & Change & $\begin{array}{l}\text { Yes (professional } \\
\text { painter) }\end{array}$ & Painting & No neuropsychological data \\
\hline $\begin{array}{l}\text { Chatterjee et al., } \\
2011 \\
\text { (Pt 2) }\end{array}$ & Stroke & $\begin{array}{l}\text { No imaging data (left } \\
\text { hemisphere stroke) }\end{array}$ & Change & $\begin{array}{l}\text { Yes (professional } \\
\text { painter) }\end{array}$ & Painting & No neuropsychological data \\
\hline $\begin{array}{l}\text { Chatterjee et al., } \\
2011 \\
(\text { Pt 3) }\end{array}$ & Stroke & $\begin{array}{l}\text { No imaging data (right } \\
\text { hemisphere stroke) }\end{array}$ & Change & $\begin{array}{l}\text { Yes (professional } \\
\text { painter) }\end{array}$ & Painting & $\begin{array}{l}\text { No neuropsychological data } \\
\text { but reports left spatial neglect }\end{array}$ \\
\hline
\end{tabular}


Table 1 | Continued

\begin{tabular}{|c|c|c|c|c|c|c|}
\hline Author and year & Diagnosis & Neuroimaging data & $\begin{array}{l}\text { Change in } \\
\text { abilities }\end{array}$ & $\begin{array}{l}\text { Previous interest in } \\
\text { Art? }\end{array}$ & $\begin{array}{l}\text { Art } \\
\text { domain }\end{array}$ & Neuropsychological data \\
\hline Takahata et al., 2014 & Stroke & $\begin{array}{l}\mathrm{CT} \text { and } \mathrm{MRI} \text { : infarction in } \\
\text { the left prefrontal region }\end{array}$ & l & Yes & Painting & $\begin{array}{l}\text { MMSE = } 26 / 30 \\
\text { Preserved visuospatial abilities } \\
\text { Deficits in episodic memory and } \\
\text { executive functions } \\
\text { Behavioral perseverations } \\
\text { No impulsiveness and no } \\
\text { compulsiveness. }\end{array}$ \\
\hline $\begin{array}{l}\text { Thomas-Anterion, } \\
2009 \\
\text { (Pt 3) }\end{array}$ & Stroke & $\begin{array}{l}\text { MRI: left posterior insula } \\
\text { and parietal operculum } \\
\text { infarct }\end{array}$ & $E$ & No & Painting & $\begin{array}{l}\text { Normal neuropsychological exam } \\
\text { Compulsive art production with } \\
\text { specific colors }\end{array}$ \\
\hline Lythgoe et al., 2005 & $\begin{array}{l}\text { Subarachnoid } \\
\text { hemorrhage }\end{array}$ & CT: no focal injury & $E$ & No & $\begin{array}{l}\text { Painting } \\
\text { Poetry } \\
\text { Sculpture }\end{array}$ & $\begin{array}{l}\text { Almost normal, except difficulties } \\
\text { in switching and inhibition control } \\
\text { Patient obsessed with art }\end{array}$ \\
\hline Galarza et al., 2014 & $\begin{array}{l}\text { Intracerebral } \\
\text { hemorrhage } \\
\text { associated to a } \\
\text { cerebral } \\
\text { arteriovenous } \\
\text { malformation }\end{array}$ & $\begin{array}{l}\text { MRI: extensive damage } \\
\text { of left temporal lobe due } \\
\text { to lobectomy. }\end{array}$ & Change & Yes & Music & $\begin{array}{l}\text { Low performance in confrontation } \\
\text { naming tests. Impairment on } \\
\text { episodic memory tests for verbal } \\
\text { modality, but not for visual } \\
\text { modality. Preserved emotion } \\
\text { recognition, except for fear. }\end{array}$ \\
\hline
\end{tabular}

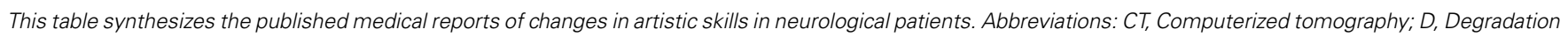

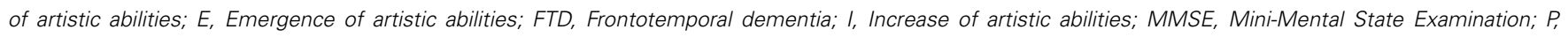

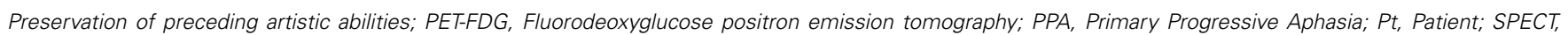

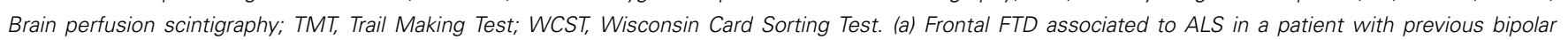

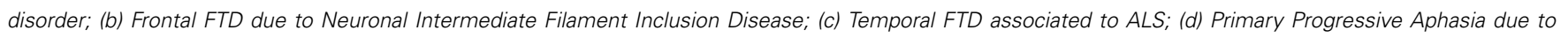
corticobasal degeneration.

10 o'clock precisely. She also presented with repetitive and ritualistic behaviors such as compulsive writing, obsessions regarding time schedules and compulsive handbag checking. In this context, the patient started to produce drawings in a compulsive manner. Mrs. YCFZ also had memory complaints, but behavioral disorders remained the most impaired domain throughout the course of the illness. Basic activities of daily living were globally preserved, although she needed assistance for some instrumental activities such as financial operations.

The standard neurological examination was normal, without eye movement disorders or extrapyramidal signs. Formal neuropsychological evaluation (November 2010-Table 3) showed an impairment in global cognitive efficiency both on the MiniMental State Exam (MMSE: 16/30; Folstein et al., 1975) and on the Mattis Dementia Rating Scale (103/144; Porto et al., 2003). Executive tasks such as DRS initiation/perseveration subscale, FAS letter fluency and digit span were altered. There was a marked episodic memory deficit, which was characterized by low performance on both learning and delayed recall tasks from the Rey Auditory Verbal Learning Test (Malloy-Diniz et al., 2007) and in the DRS memory subscale. There was a moderate impairment in the naming task (9/15; Bertolucci et al., 2001). The visual abilities assessed using the Visual Object and Space Perception Battery (Warrington and James, 1991; Quental et al., 2013) were preserved (number location and cube analysis). The patient had no deficit on gesture execution, and no signs of Balint or Gertsmann syndromes. Brain computed tomography scanning in 2009 showed a remarkable atrophy in temporopolar regions bilaterally and a mild frontal polar atrophy (Figure 1). Brain MRI performed 2 years later showed no signs of cerebrovascular disease and confirmed the same regional atrophy pattern with additional diffuse brain shrinkage. On clinical follow-up after 36 months, the global cognitive efficiency assessed using MMSE remained stable (see Table 4), although language and functional abilities deteriorated, as assessed using the Functional Activities Questionnaire (Pfeffer et al., 1982). The diagnosis of probable bvFTD was retained on a clinical basis.

The patient was never notably interested in art. However, during the course of her disease, she began to draw compulsively on a daily basis (Figure 2). We sought to systematically analyze her drawing production using independent tools for this assessment. For this purpose, we used the consensual assessment technique (CAT; Amabile, 1982) to measure the global creativity of each drawing combined with a questionnaire adapted from Drago and colleagues (Drago et al., 2006a). The criteria assessed in this questionnaire included "Aesthetics": How beautiful is the painting? "Closure": How complete is the painting? "Abstraction": How abstractive is the painting? "Obsessions/Repetition": How obsessive/repetitive is the painting? "Evocative Impact": How strongly does the painting induce feelings or thoughts? "Novelty": How 
Table 2 | Synthesis of cognitive, behavioral and artistic changes in previous published cases of patients listed in Table 1 and in our patient.

\begin{tabular}{|c|c|c|c|c|c|}
\hline Pathology & Bv-FTLD & Temp-FTLD & nfPPA-FTLD & Alzheimer's disease & Other \\
\hline Number of patients & 11 & 19 & 4 & 8 & 12 \\
\hline Artistic emergence & 5 & 11 & 0 & 1 & 2 \\
\hline Artistic increase (or preservation) & $4(1)$ & $2(6)$ & $2(1)$ & $1(1)$ & $4(3)$ \\
\hline Artistic degradation & 1 & 0 & 1 & 5 & 3 \\
\hline Artistic domain = visual & 10 & 8 & 4 & 8 & 11 \\
\hline
\end{tabular}

Intact visuospatial abilities

7 out of 7 reported 9 out of 10 reported 3

The degradation of artistic skills was associated with impaired visuospatial abilities in 6 cases out of 8 reported

Positive behavioral symptoms reported

Perseverations

Disinhibition

Compulsions obsessions

Negative dysexecutive symptoms reported

1 or several deficits

Abstraction difficulties

$\begin{array}{ll}3 & 2 \\ 5 & 7 \\ 9 & 5\end{array}$

10 out of 10 reported 7 out of 9 reported

5 out of 8 reported

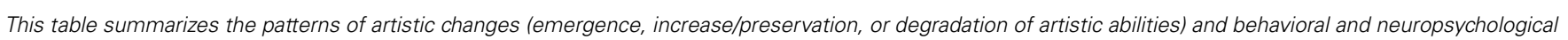

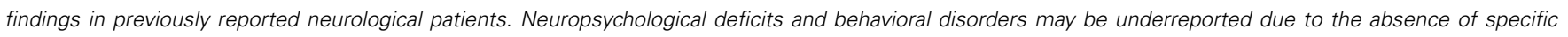

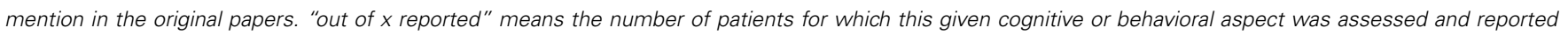

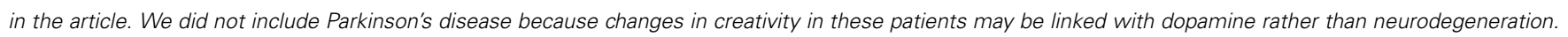

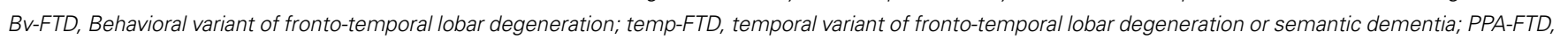

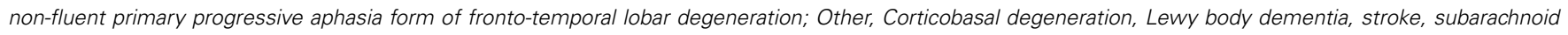
hemorrhage and cerebral arteriovenous malformation.

Table 3 | Neuropsychological assessment of the patient YCFZ (November 2010).

Test

Patient score Standard deviation

\begin{tabular}{lcc}
\hline MATTIS TOTAL SCORE (/144) & $\mathbf{1 0 3}$ & $\mathbf{- 9 . 4 7}$ \\
MATTIS-Attention (/37) & 33 & -1.83 \\
MATTIS-Initiation (/37) & 19 & -4.26 \\
MATTIS-Construction (/6) & 6 & \\
MATTIS-Concepts (/39) & 37 & \\
MATTIS-Memory (/25) & 8 & -2.59 \\
Verbal SPAN (DIRECT-INDIRECT) & $\mathbf{4 - 3}$ &
\end{tabular}

Rey auditory verbal learning test

$$
\text { Immediate recall list } A
$$

Delayed recall list $A$

Recognition test list $A$

NAMING (BOSTON-CERAD) (/15)

FAS-Total

Letter $F$

Letter A

Letter S

\section{VISUAL OBJECT AND SPACE PERCEPTION}

Number location (/20)

Cube analysis (/20)

${ }^{*}$ This cut-off distinguished controls from patients with early Alzheimer's disease with 63\% sensitivity and $74 \%$ specificity (Quental et al., 2013).

${ }^{\S}$ This cut-off distinguished controls from patients with early Alzheimer's disease with $75 \%$ sensitivity and 68\% specificity (Quental et al., 2013).

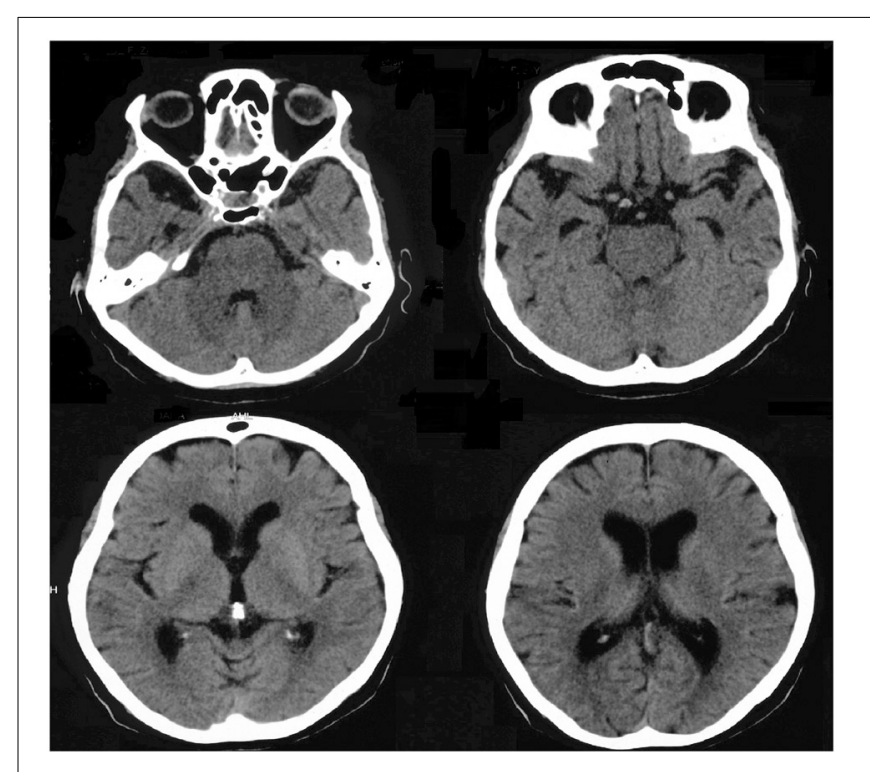

FIGURE 1 | Brain computed tomography scan performed in 2009 showing marked atrophy in bilateral temporopolar and frontal regions.

original or new is the painting? "Representation": How well is the subject of the painting rendered? "Technique": How much skill does the painting demonstrate?

We selected 20 drawings from May 2010 to September 2013 and asked 12 independent professional visual artists from Brazil (5 men, 7 women, aged from 31 to 70 years old, 5 of which 
Table 4 | Longitudinal cognitive assessment of Mrs YCFZ, from November 2010-September 2013.

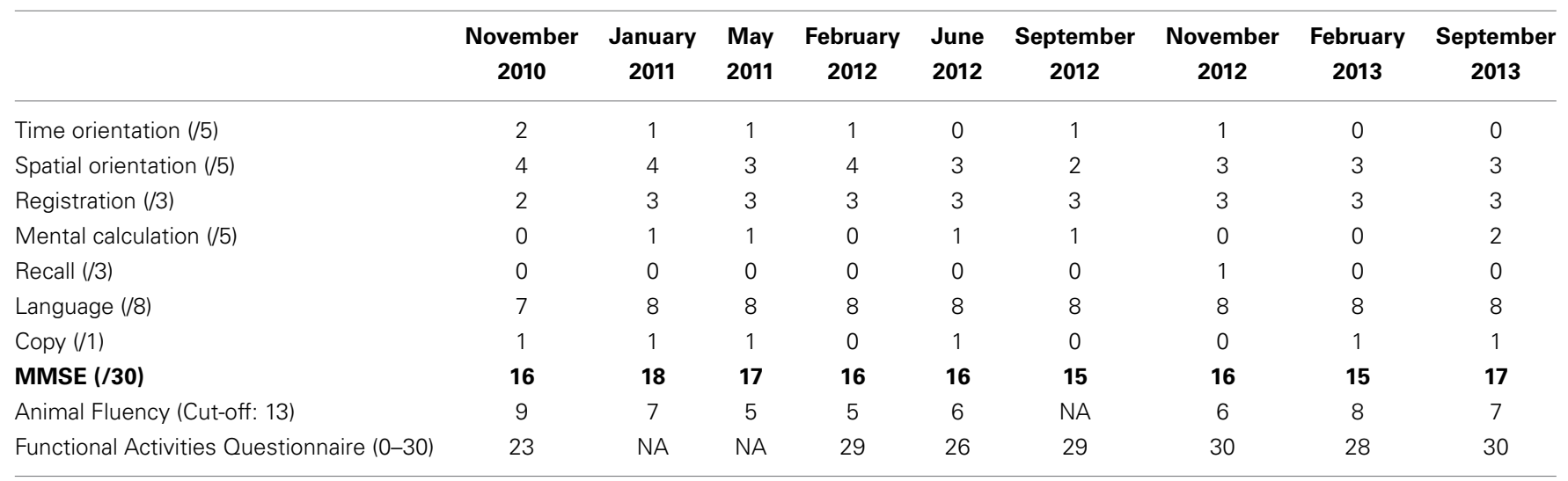

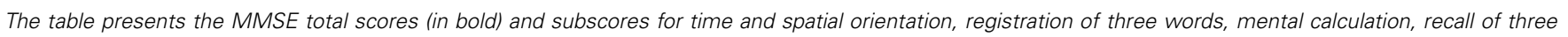

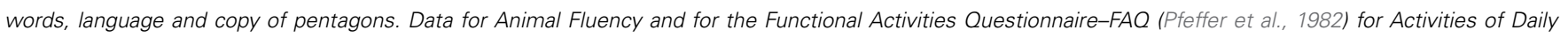
Living are also presented. A cut-off point higher than 9 in the FAQ indicates impaired function and cognitive impairment. (NA, Not available).

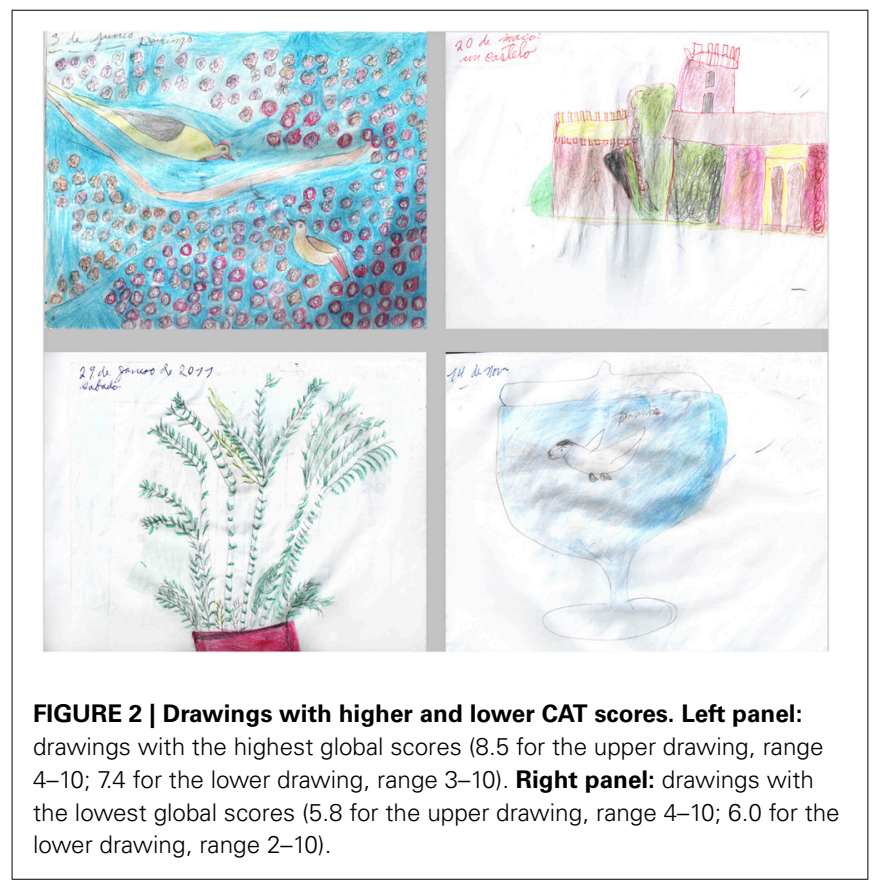

were professors at Fine Art universities, most of which had formal artistic training in Fine Arts) to judge the drawings according to global creativity and the criteria explored in the questionnaire. The experts were also encouraged to make free comments. This expert group was blind to the clinical condition of the patient, and no information on her artistic status or training was given.

The results of this evaluation are presented in Table 5. The mean global creativity score across experts and drawings was 6.6, but varied markedly depending on the expert, ranging from 3.2 to 9.6. Scores for each criterion also showed a considerable heterogeneity between the experts ranging from 0 to 10 for each drawing. This heterogeneity suggests that the 12 scorers, all experts in the domain of visual arts, had a different conception of what creativity and its related features should be.
CAT does not give an absolute assessment of creativity but provides relative scores enabling the comparison between different productions or different groups of participants. Therefore, we attempted to evaluate the evolving profile of the patient's drawings across time periods. First, we pooled drawings performed each year from 2010 to 2013 and looked at scores across the years (Figure 3). We observed an increase in scores from the first drawings (2010) to the last drawings (2013) in all of the evaluated aspects. Then, we statistically compared two periods: an early (drawings from 2010 and $2011 ; n=8$ ) and a late period (those from 2012 and 2013; $n=12$ ) using a non-parametric Wilcoxon signed rank test. An increase in creativity scores was statistically significant for abstraction $(Z=-2.756, p=0.006)$, obsession $(Z=-2.045, p=0.041)$ and novelty $(Z=-2.312$, $p=0.021$ ) subscores (Figure 3).

In their free comments, expert artists mentioned that most of the drawings were beautiful and creative, drawn with care, and found the compositions interesting or original. They insisted on the "naïve" character of the drawings, frequently describing them as simple and infantile ("these drawings are similar to those from my daughter of 6 years of age," translated general comment from expert 1). The experts agreed on the representational rather than abstract nature of the productions. Repetitions, obsessions, or stereotypies were diversely interpreted. Many experts highlighted the repetitive and obsessive character of the drawings, but they often found them useful for the composition, the expression, or the rhythm of the picture, and gave low obsession scores for this reason. There was a large variability in the scores for repetitions and obsessions (minimal 0, maximal 9.1, with a mean of 5.2). The drawings were often described as expressive and containing negative emotions ("sinister paranoid atmosphere," translated from expert 8 about drawing 19), but harmony was also evoked for some of them. Other comments highlighted bizarre or interesting compositions or strange/poor color choices.

Overall, the quantitative and qualitative creativity assessments showed great heterogeneity, especially in the general creativity of the drawings, the role of repetitions in the composition, or the emotional content. The disparity of judgment between 
Table 5 | CAT assessment of the drawings from patient YCFZ (2010-2013).

\begin{tabular}{|c|c|c|c|c|c|c|c|c|c|}
\hline & $\begin{array}{l}\text { Global } \\
\text { Score }\end{array}$ & Aesthetics & Closure & Abstraction & $\begin{array}{l}\text { Obsessions/ } \\
\text { repetitions }\end{array}$ & $\begin{array}{l}\text { Evocative } \\
\text { impact }\end{array}$ & Novelty & Representation & Technique \\
\hline Artist 1 & 4.8 & 4.4 & 5.7 & 4.1 & 4.1 & 3.8 & 3.6 & 4.7 & 3.3 \\
\hline Artist 3 & 3.2 & 0.3 & 9.2 & 1.5 & 8.5 & 0.3 & 0.9 & 3.0 & 0.0 \\
\hline Artist 4 & 9.4 & 9.1 & 9.4 & 9.1 & 9.1 & 9.8 & 9.3 & 9.8 & 9.6 \\
\hline Artist 7 & 9.3 & 8.9 & 9.2 & 1.3 & 5.3 & 8.2 & 8.4 & 8.7 & 8.8 \\
\hline Artist 8 & 3.2 & 3.0 & 3.1 & 3.3 & 4.6 & 3.6 & 3.2 & 3.0 & 2.9 \\
\hline Artist 9 & 7.0 & 5.6 & 7.7 & 3.9 & 4.5 & 6.2 & 6.5 & 7.5 & 6.5 \\
\hline Artist 10 & 7.9 & 7.2 & 8.5 & 7.5 & 3.7 & 7.6 & 7.8 & 7.3 & 6.5 \\
\hline Artist 11 & 6.8 & 5.7 & 8.4 & 1.7 & 5.4 & 6.1 & 3.8 & 7.4 & 5.6 \\
\hline Max & 9.6 & 9.5 & 10.0 & 9.1 & 9.1 & 9.8 & 10.0 & 9.8 & 10.0 \\
\hline
\end{tabular}

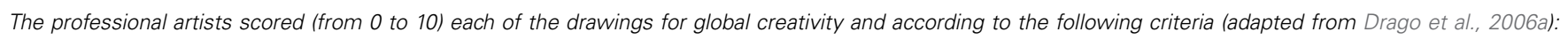

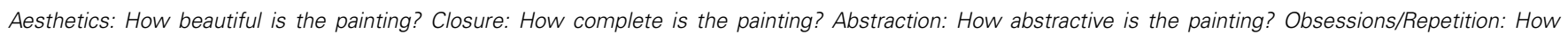

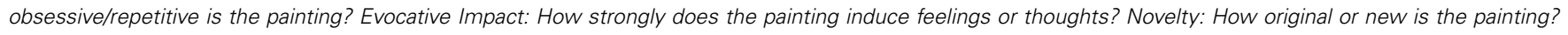

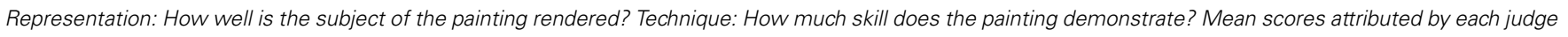
to the 20 assessed drawings are provided together with standard deviation, minimum and maximum values (in bold).

the professional artists with academic training for most indicates that personal subjectivity strongly influenced the scoring. Despite a large inter-judge variability, an improvement of the patient's artistic skills was considered during a 3-year evolution period, especially for the abstraction, novelty, and repetition criteria, while language and autonomy declined. This suggests that the artistic creative capacity of the patient did not parallel her cognitive deterioration.

This observation is consistent with the potential emergence of an artistic inclination during the evolution of bvFTD, as previously reported, and highlights the interference between cognitive and behavioral frontal symptoms and creative production.

\section{DISCUSSION: WHAT DO ARTISTIC PATIENTS TELL US ABOUT CREATIVITY?}

The difference between controlled patient studies and medical reports of creativity following frontal damage raises interesting questions regarding the mental components of creative thinking, their measurements, and their neural bases. Experimental approaches of creativity have demonstrated that various PFC regions are critical to creative capacity. Conversely, some frontal patients exhibit new or significant artistic productions despite their frontal dysfunctions, as was the case for the reported patient. Can this be explained? Does this mean that their creative capacities increased?

\section{CLINICAL CONSIDERATIONS FOR PATIENTS WITH NEW OR SIGNIFICANT ARTISTIC PRODUCTION}

Artistic facilitation is a rare phenomenon in neurological patients. The link between artistic production and neurological diseases appears to be anecdotal, especially when the high incidence of strokes and neurodegenerative diseases are considered. SD (FTD with temporal prominent atrophy) is the most frequent diagnosis associated with increased creative production (Table 2). In contrast with controlled studies that included unselected patients with neurodegenerative diseases, case reports point to particular patients who are especially concerned with making art. To our knowledge, no such exceptional patient with artistic facilitation has been explored using theory-based creativity tests. So it cannot be ruled out that controlled studies with unselected patients may miss some exceptional patients.

Because artistic facilitation has been observed in diseases as different as temporal and frontal variants of FTD, Alzheimer's disease, or stroke affecting distinct brain regions, clinical reports do not argue for a specific neuroanatomical or neuropsychological pattern associated with enhanced artistic production. For instance, it has been proposed that the emergence of artistic talent in FTD patients results from the release of the inhibition exerted by anterior regions over the posterior regions involved in visuospatial processing (Kapur, 1996; Mendez, 2004; Seeley et al., 2008). This theory may not explain the improved or preserved creative output in patients with predominant posterior injury (Annoni et al., 2005; Fornazzari, 2005) or in patients with no frontal dysfunction (Schrag and Trimble, 2001; ThomasAnterion et al., 2010). Nevertheless, it is remarkable that most positive changes of artistic abilities concerned visual arts (41 cases out of 54 reviewed, including the current case report) when most patients had preserved visuospatial skills each time this was reported. In the related cases of creative production associated with either bvFTD or SD, degeneration mostly affected 
the left temporal and/or frontal regions, which may explain the predominance of visual arts in the patients' production being more related to the visuospatial functions of the right hemisphere. However, Wu and colleagues (Wu et al., 2013) recently reported two SD patients in whom the emergence of artistic activities in the verbal domain was associated with a predominantly left atrophy. Additionally, this left-right hypothesis is not in agreement with functional imaging data, as the metaanalysis from Gonen-Yaacovi and colleagues demonstrated a left dominance of activation in both verbal and visual tasks. Unfortunately, most of the published clinical reports do not provide extensive or detailed neuropsychological and anatomical data, which would enable a better characterization of the relationship between frontal or visuospatial alterations and creative output.

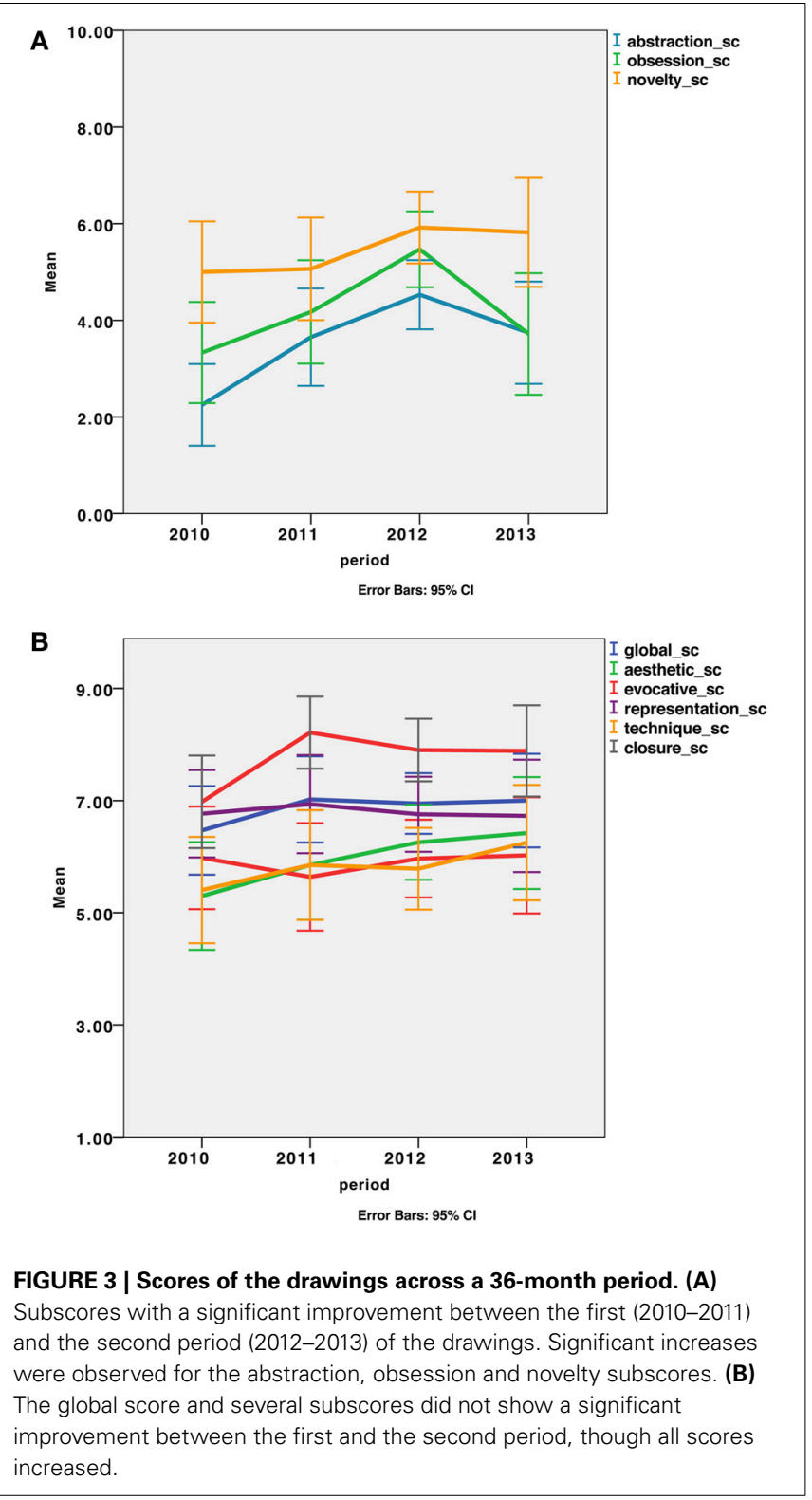

\section{TIES BETWEEN FRONTAL SYMPTOMS AND ARTISTIC PRODUCTIONS}

Some behavioral disorders associated with frontal damage may account for or parallel artistic expression, as suggested previously (Rankin et al., 2007; de Souza et al., 2010; Palmiero et al., 2012; Schott, 2012) and highlighted in our reported case. From a neurological point of view and based on the neuropsychological profile of our patient, we fist concluded that some frontal symptoms are possibly interfering with the drawings, while preserved visuospatial abilities enable their execution. The urge to draw on a daily basis and the huge amount of productions are possibly related to personality changes and compulsive behaviors provoked by frontal damage. Repetitive topics (plants, animals, people) and patterns (volcano, leaves) may be the manifestation of perseverations and stereotypies due to the frontal syndrome. Strange composition and infantile features may be explained by poor planning abilities (Figure 4).

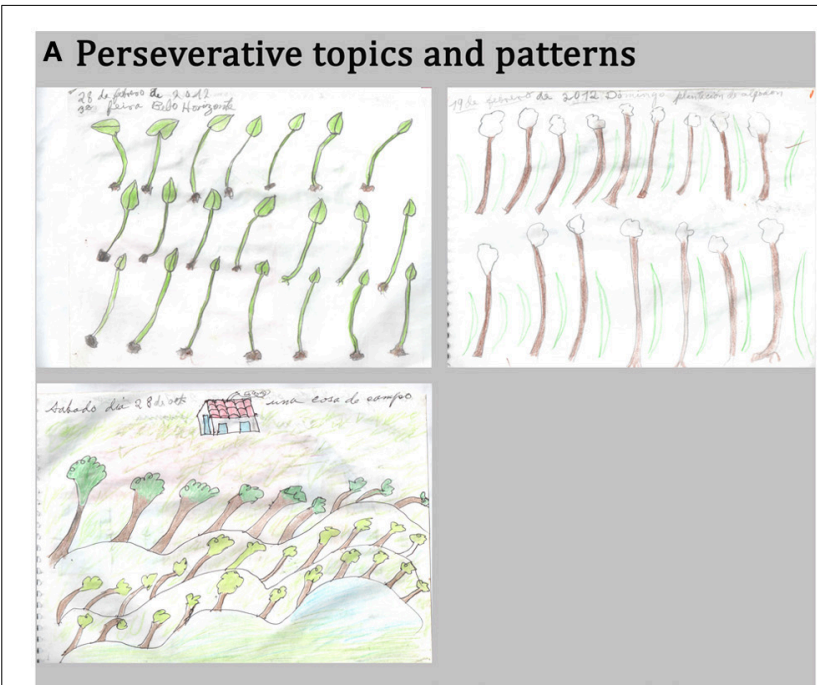

\section{B Strange composition/colors, simplifications}

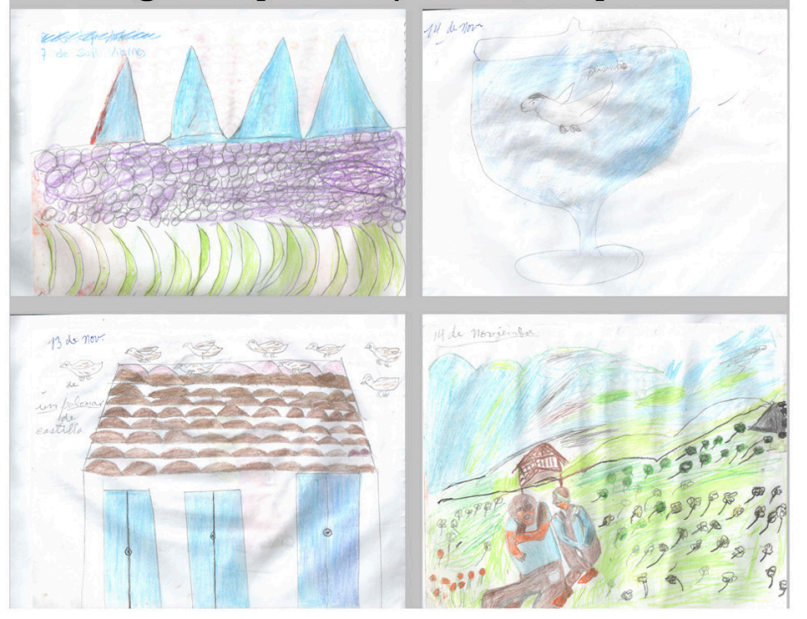

FIGURE 4 | Possible frontal manifestations expressed in the patient's drawings. (A) Perseverative topics and patterns. (B) Strange composition, color choices and simplifications. 
Many patients with so called "artistic improvement" presented compulsive and/or obsessive behaviors (Finkelstein et al., 1991; Miller et al., 1998; Miller and Hou, 2004; Lythgoe et al., 2005; Serrano et al., 2005; Thomas-Anterion et al., 2010). As pointed by Schott (2012), in such patients, "a strong preference for a single art medium, a restricted focus on artistic themes, repetition, compulsion and seeking for perfection (...) enabled remarkable artistry to be achieved." The patient we report on also produced drawings in a compulsive manner; this may partly account for the acquisition of an artistic technique. The fact that her last drawings received higher scores than the first drawings (produced 3 years prior) supports this assumption. Compulsive and/or obsessive behaviors are a major symptom of bvFTD (Rascovsky et al., 2011). These behaviors are surprisingly in contrast with the apathy also frequently observed in bvFTD, as well as with the cognitive inertia associated with a poor fluency, as was the case in our patient. Compulsive behaviors are usually associated with severe disorders of social conduct. For example, the patient reported by Miller et al. (1998) developed new photographic skills during the course of FTD. Pictures were taken compulsively to obtain a "perfect image." However, at the same time, this compulsive demeanor also produced socially inappropriate behaviors, leading to severe social constraints, and ultimately to institutionalization. The patient we report also had ritualized behaviors that also led to social misconduct. In other words, the repetitive and ritualized behaviors related to frontal dysfunction may be expressed in the artistic domain, leading to new interests in making art or intense artistic activity with repetitive topics or productions. The reasons why some patients focus their compulsive behaviors on making art and others do not remain poorly understood.

Perseverations or patterning, which are also linked to frontal damage, were observed in our patient's drawings (trees and leaves, for instance). Surprisingly, our expert group remarked repetitions and made free comments about them but did not give especially high scores on the repetition criteria because they did not feel it was inappropriate or unaesthetic. A previous case-control study of creative production across bvFTD patients and normal controls (de Souza et al., 2010) showed that behavioral disorders, such as perseverations, may also partly explain the "originality" of some productions when frontal patients perform divergent thinking tests, but overall their originality score was impaired. Similarly, disinhibition, another cardinal symptom of frontal dysfunction, can interfere with creative activities, as also noted by de Souza et al. (2010); however this was not observed in the current case. Social disinhibition can lead to unexpected choices of topics, for instance with sexual content. The release of the inhibition exerted by frontal regions over the posterior regions may explain some unconventional or socially unusual aspects of creative productions as well as behaviors in frontal patients (Miller et al., 1996; Mell et al., 2003; Mendez, 2004; Miller and Hou, 2004; Drago et al., 2006b; Seeley et al., 2008).

In the cognitive sphere, some frontal lesions may help in overcoming knowledge constraints (Reverberi et al., 2005; Abraham, 2014). Patients with lateral prefrontal damage may experience a less sculpted (less constrained) response space in a given context than healthy subjects, enabling them to more easily consider any option, including those outside of contextual constraints
(Reverberi et al., 2005). Overall, disinhibition or the loss of social conventions and associative knowledge may allow the emergence of creative productions (Miller et al., 1996, 2000; Miller and Hou, 2004; Liu et al., 2009). According to Rankin et al. (2007), productions from bvFTD patients may have an artistic value in the sense that they are freer from conventional representations and social conventions about art. It is more difficult to assume that this freeing from convention is an intentional and voluntary act.

Finally, our patient's drawings share other qualitative features that have been reported in previous FTD patients, especially with those described in Rankin and colleagues' study (Rankin et al., 2007) in which patient productions were assessed by scientists who had an interest in arts and not by professional artists. For instance, landscapes, people, animals and plants appear to be the preferred topics in frontal patient's productions. These preferred topics may be considered to be conventional and concrete but are often represented in an unusual way. The simplification of representations, judged as naïve or infantile, and unconventional or disordered composition with eccentricity of the subject, could be linked to a poor planning ability and lack of abstraction but could also contribute to the bizarreness and unusualness of the drawings.

Together, patient observations indicate that some clinical and behavioral symptoms of frontal dysfunction may facilitate the appearance of creative features in artistic products. This explanation cannot stand in the domain of creativity in which other frontal functions such as cognitive control, planning, mental manipulation, and abstraction are critical. Additionally, these observations raise the question of whether the artistic productions we observe reflect the same aspect of creative capacity and result from the same voluntary creative processes that are assessed in experimental creativity studies.

\section{ARTISTIC AND NEUROSCIENTIFIC PERSPECTIVES}

Patient studies and clinical observations may highlight the probable difference between creativity evaluated from an artistic point of view and creativity evaluated from a neuroscientific perspective. In the field of Art, aspects such as emotional or evocative impact, provocation and message, aesthetic value, or technical mastery may be more important than in other domains such as sciences and technology. These aspects are not captured by the consensual definition of creativity that focuses on originality and appropriateness. Within the frame of this definition, a difference may also be noted: originality may often be considered to be a predominant condition for creativity in the artistic field in which appropriateness is difficult to apprehend; however, in other domains such as science, appropriateness is a requirement. For example, patients studied in de Souza et al. (2010) were often inappropriate in their responses, while no control subjects were. The sexual content of their drawings may be regarded as inappropriate in an experimental testing context but is usually well accepted in artistic works. This suggests that each domain of creative expression differently prioritizes originality and appropriateness and makes different demands on the mental operations to achieve them.

It is also important to mention that experimental and neuroimaging approaches do not assess motivational, conative, or 
emotional factors affecting creative drive. However, these factors appear to be important in real life creativity, such as in the spontaneous productions of patients with an artistic preoccupation. As highlighted by Schott (2012), these patients are often described "as obsessive about their art, with an urge to create." It is then possible that emotional and motivational factors play an important role in real life creativity, including artistic creativity, but are poorly accounted for in experimental approaches of creativity. The latter are indeed based on cognitive theories of creative capacity with limited assessment of the emotional and conative aspects.

In our case, the score of evocative impact, which is intended to depend on the emotional expressiveness of the drawings, varied between the experts (0.3-9.8, with a mean value of 5.4). One method for analyzing the importance of emotional process in the artistic production of the patient would be to study the correlations between the scores on the CAT and objective measures of social-emotional cognition such as emotion recognition, empathy, and theory of mind. Unfortunately, these domains were not evaluated in our patient.

Finally, the difference between real life and experimental settings for measuring creativity is also in question. The evaluation of spontaneous patient productions was generally based on subjective assessments from authors, experts, or groups of judges. Our clinical case illustrates that subjective assessment, although framed by determined criteria and performed by experts in the field of visual arts, has a great inter-individual variability. Experimental theory-based approaches use more objective criteria to measure creative capacity, for instance fluency, flexibility, originality, or problem solving success. Several of these cognitive approaches have been used to study the neural basis of creativity in functional neuroimaging and in neurological patients. If theory-based approaches use more "objective" criteria, they are constrained by the hypothesis that they rely on. In other words, creativity tasks only assess the processes involved in creative capacity according to the cognitive model used. Each theorybased approach focuses on one or more aspects of the creative process, but none of them evaluates the creativity in all of its dimensions. In particular, theory-based creativity tasks do not necessarily capture artistic quality, even though they have been shown to be ecologically valid and statistically linked with artistic creativity (Kim, 2006; Plucker and Makel, 2010). On the contrary, theory-free creativity assessments, such as CAT, are not based on any particular theory of creativity, which means that their validity is not dependent upon the validity of any particular theory. Unfortunately, our patient was no longer able to perform experimental creativity tests at the time of the consultation; thus, we are not able to compare both approaches to creativity assessment in a case of artistic preoccupation.

Overall, the creativity attributed to patients preoccupied with arts during a frontal disease and creativity explored in experimental studies differ in several conceptual and experimental ways, and are probably affected differently by frontal symptoms.

\section{OVERALL, CAN HYPOFRONTALITY FACILITATE CREATIVITY?}

A common notion suggests that losing control, especially relaxing social and emotional inhibitions or conventions, may favor personal expression and creativity. The use of drugs such as alcohol may aim to approach this state. Several artistic streams are based on the spontaneous, non-controlled generation of ideas or objects, for instance using automatic writing or random painting. In the neuroscientific literature, some studies suggest that unconscious and uncontrolled processes facilitate divergent thinking and insight problem solving (Yaniv and Meyer, 1987; Dijksterhuis and Meurs, 2006; Dorfman et al., 2008; Zhong et al., 2008; Ritter and Dijksterhuis, 2014). Because control in behavioral, affective, social and cognitive spheres is largely associated with the functions of the PFC, the notion that hypofrontality could favor creativity may be valid. A recent theory (Chrysikou et al., 2013) also postulates that hypofrontality may enhance some aspects of creativity: the availability of bottom-up information that is usually filtered by the PFC may favor a breaking away from rule-based thinking. This is reinforced by the fact that some frontal patients appear to have abilities in some aspects of artistic expression.

The current review identified several clinical aspects of hypofrontality in the social, conative and cognitive domains that could explain some creative features of the patients' products. First, a social aspect related to the common view of hypofrontality is disinhibition. Social disinhibition can lead frontal patients to break with social conventions and propose unusual productions in creative (but also in uncreative) activities. Second, compulsive, repetitive or obsessive behaviors may lead to high productivity and improvements in technical skills. This obsessive-compulsive trait acts as a strong motivation toward a given activity. A third and more paradoxical aspect consists of a lower influence of habitual contextual associations in frontal patients. Patients with lateral PFC damage may be less constrained by learned rules, which may facilitate some problem-solving tasks (Reverberi et al., 2005). This aspect is paradoxical because it is in apparent opposition to the acknowledged role of the inferolateral PFC in overcoming prepotent responses [as observed in functional imaging and patient studies using Stroop tasks, no-go tasks or Hayling tasks (Aron et al., 2003; Brass et al., 2005; Picton et al., 2007; Volle et al., 2012), as well as in contextual control (Azuar et al., 2014)]. Overcoming prepotent responses and contextual control are both thought to play a role in creativity. Thus, whether highly creative people among the general population have more relaxed contextual constraints (as frontal patients may have) or increased abilities to intentionally overcome these constraints is an interesting topic for future research.

If hypofrontality generally evokes signs of disinhibition and poor control (usually associated with lateral and ventral portions of the PFC), we should also consider other roles of PFC in cognition that have been more recently highlighted. For instance, the medial PFC is part of the default network (Buckner et al., 2008), a set of functionally connected brain regions in which activity decreases when tasks require more focal attention, effort, or control. This network has been associated with spontaneous cognition and mind wandering (Gilbert et al., 2007; Mason et al., 2007; Christoff et al., 2009; AndrewsHanna et al., 2010). This network can be distinguished from the set of regions functionally connected to lateral PFC (Gilbert et al., 2010). Some recent studies highlighted the role of the default network and of spontaneous cognition in creativity 
(Takeuchi et al., 2012; Wise and Braga, 2014). Medial PFC is also involved in semantic processing (Buckner et al., 2008; Binder et al., 2009) and in semantic aspects of creativity (Green et al., 2012; Abraham, 2014). These data suggest that the lateral PFC is engaged in rule-based thinking, while the medial PFC appears to be involved in a more spontaneous mode of thinking such as associative thinking. The rostral PFC may act as a switch (Burgess et al., 2007) between these two modes. Both thinking modes are thought to be required for creativity, as suggested in several models (Vartanian et al., 2007; Gabora, 2010; Ward and Kolomyts, 2010; Ellamil et al., 2012). For instance, the uncontrolled association of ideas triggered by perceptual or emotional stimuli may favor unusual responses but may also lead to inappropriate responses if the control mode does not filter. How each mode is affected by frontal lesions and how it impacts creative capacity is poorly known. The consequences of damage to the rostromedial PFC on creativity may lead to poorer originality (de Souza et al., 2010; Shamay-Tsoory et al., 2011), but the mechanisms of this change and its relationship with the default network functions are unexplored.

Overall, the classical view in which PFC supports top-down controlled processing while subcortical and posterior brain regions are engaged in bottom-up uncontrolled processing may be more balanced regarding creativity. If the lateral PFC is largely associated with top-down control, some other PFC regions may be involved in the uncontrolled or bottom-up processing that is spontaneous cognition, including semantic associations and mind wandering. The interaction between controlled and spontaneous cognition via connectivity between the lateral and medial PFC networks (Spreng et al., 2010) may enable both original and appropriate ideas to emerge.

\section{CONCLUSION}

The functional and anatomical organization of the PFC supports different aspects of behavioral adaptation in humans, suggesting its role in the adaptive aspects of creativity as they are emphasized in its definition (i.e., creating something original and appropriate). Functional neuroimaging and experimental patient studies also suggest that the PFC, in particular the anterior PFC, may also play a critical role in originality aspects of creativity. Damage to the PFC may alter the intentional appropriateness and originality of patient productions by altering planning, fluency, mental flexibility, rule-based thinking, or abstraction. However, clinical observations of frontal damage patients suggest that some symptoms associated with frontal damage provoke cognitive, conative, and behavioral changes, including social disinhibition, compulsive behaviors, emotional distortions, and the relaxing of cognitive constraints, which can motivate and favor artistic productions. However, artistic production is not synonymous with creativity, because creativity refers to aspects such as emotional expression, evocative impact, aesthetic, and technical abilities, which are present in art but not necessarily in other domains of creativity. Art is thus difficult to capture using theory-based creativity tasks, and to our knowledge, patients with facilitation in the artistic domain have not been tested experimentally with such tasks. Therefore, whether these rare frontal patients increase their real creative capacity does not have a yes or no answer. Using theory-based creativity tasks, functional imaging and patient data suggest that distinct PFC subregions differently affect the different aspects of creativity. PFC cannot be considered as a unitary structure, and exploring its organization and interactions subserving different creativity processes, including controlled and spontaneous cognition, as well as social and affective aspects, may provide a more precise answer.

\section{ACKNOWLEDGMENTS}

This work was supported by the National Research Agency (ANR09-RPDOC-004-01; Emmanuelle Volle) and by The National Council for Scientific and Technological Development (Brazil, CNPq-402853/2012-1; Leonardo C. de Souza). We thank our patient, Mrs. YCFZ, and her family for their willingness and interest in our research. We also thank all of the artists who evaluated the drawings of Mrs. YCFZ.

\section{REFERENCES}

Abraham, A. (2014). Creative thinking as orchestrated by semantic processing vs. cognitive control brain networks. Front. Hum. Neurosci. 8:95. doi: 10.3389/fnhum.2014.00095

Abraham, A., Beudt, S., Ott, D. V. M., and Yves von Cramon, D. (2012). Creative cognition and the brain: dissociations between frontal, parietaltemporal and basal ganglia groups. Brain Res. 1482, 55-70. doi: 10.1016/j.brainres.2012.09.007

Amabile, T. M. (1982). Social psychology of creativity: a consensual assessment technique. J. Pers. Soc. Psychol. 43, 997-1013. doi: 10.1037/0022-3514.43.5.997

Andrews-Hanna, J. R., Reidler, J. S., Huang, C., and Buckner, R. L. (2010). Evidence for the default network's role in spontaneous cognition. J. Neurophysiol. 104, 322-335. doi: 10.1152/jn.00830.2009

Annoni, J. M., Devuyst, G., Carota, A., Bruggimann, L., and Bogousslavsky, J. (2005). Changes in artistic style after minor posterior stroke. J. Neurol. Neurosurg. Psychiatr. 76, 797-803. doi: 10.1136/jnnp.2004.045492

Arden, R., Chavez, R. S., Grazioplene, R., and Jung, R. E. (2010). Neuroimaging creativity: a psychometric view. Behav. Brain Res. 214, 143-156. doi: 10.1016/j.bbr.2010.05.015

Aron, A. R., Fletcher, P. C., Bullmore, E. T., Sahakian, B. J., and Robbins, T. W. (2003). Stop-signal inhibition disrupted by damage to right inferior frontal gyrus in humans. Nat. Neurosci. 6, 115-116. doi: 10.1038/ nn 1003

Asari, T., Konishi, S., Jimura, K., Chikazoe, J., Nakamura, N., and Miyashita, Y. (2008). Right temporopolar activation associated with unique perception. Neuroimage 41, 145-152. doi: 10.1016/j.neuroimage.2008.01.059

Aziz-Zadeh, L., Kaplan, J. T., and Iacoboni, M. (2009). "Aha!": the neural correlates of verbal insight solutions. Hum. Brain Mapp. 30, 908-916. doi: 10.1002/hbm.20554

Aziz-Zadeh, L., Liew, S.-L., and Dandekar, F. (2012). Exploring the neural correlates of visual creativity. Soc. Cogn. Affect. Neurosci. 8, 475-480. doi: 10.1093/scan/nss021

Azuar, C., Reyes, P., Slachevsky, A., Volle, E., Kinkingnehun, S., Kouneiher, F., et al. (2014). Testing the model of caudo-rostral organization of cognitive control in the human with frontal lesions. Neuroimage 84, 1053-1060. doi: 10.1016/j.neuroimage.2013.09.031

Badre, D., and Wagner, A. D. (2007). Left ventrolateral prefrontal cortex and the cognitive control of memory. Neuropsychologia 45, 2883-2901. doi: 10.1016/j.neuropsychologia.2007.06.015

Bechtereva, N. P., Korotkov, A. D., Pakhomov, S. V., Roudas, M. S., Starchenko, M. G., and Medvedev, S. V. (2004). PET study of brain maintenance of verbal creative activity. Int. J. Psychophysiol. 53, 11-20. doi: 10.1016/j.ijpsycho.2004.01.001

Bengtsson, S. L., Csikszentmihalyi, M., and Ullen, F. (2007). Cortical regions involved in the generation of musical structures during improvisation in pianists. J. Cogn. Neurosci. 19, 830-842. doi: 10.1162/jocn.2007.19.5.830

Berkowitz, A. L., and Ansari, D. (2008). Generation of novel motor sequences: the neural correlates of musical improvisation. Neuroimage 41, 535-543. doi: 10.1016/j.neuroimage.2008.02.028 
Bertolucci, P. H., Okamoto, I. H., Brucki, S. M., Siviero, M. O., Toniolo Neto, J., and Ramos, L. R. (2001). Applicability of the CERAD neuropsychological battery to Brazilian elderly. Arq. Neuropsiquiatr. 59, 532-536. doi: 10.1590/S0004282X2001000400009

Binder, J. R., Desai, R. H., Graves, W. W., and Conant, L. L. (2009). Where is the semantic system? A critical review and meta-analysis of 120 functional neuroimaging studies. Cereb. Cortex 19, 2767-2796. doi: 10.1093/cercor/bhp055

Bogousslavsky, J. (2005). Artistic creativity, style and brain disorders. Eur. Neurol. 54, 103-111. doi: 10.1159/000088645

Brass, M., Derrfuss, J., Forstmann, B., and von Cramon, D. Y. (2005). The role of the inferior frontal junction area in cognitive control. Trends Cogn. Sci. 9, 314-316. doi: 10.1016/j.tics.2005.05.001

Buckner, R. L., Andrews-Hanna, J. R., and Schacter, D. L. (2008). The brain's default network: anatomy, function, and relevance to disease. Ann. N.Y. Acad. Sci. 1124, 1-38. doi: 10.1196/annals.1440.011

Budrys, V., Skullerud, K., Petroska, D., Lengveniene, J., and Kaubrys, G. (2007). Dementia and art: neuronal intermediate filament inclusion disease and dissolution of artistic creativity. Eur. Neurol. 57, 137-144. doi: 10.1159/000098464

Burgess, P. W., Alderman, N., Volle, E., Benoit, R. G., and Gilbert, S. J. (2009). Mesulam's frontal lobe mystery re-examined. Restor. Neurol. Neurosci. 27, 493-506. doi: 10.3233/RNN-2009-0511

Burgess, P. W., Dumontheil, I. I., and Gilbert, S. J. (2007). The gateway hypothesis of rostral prefrontal cortex (area 10) function. Trends Cogn. Sci. 11, 290-298. doi: 10.1016/j.tics.2007.05.004

Cardillo, E. R., Watson, C. E., Schmidt, G. L., Kranjec, A., and Chatterjee, A. (2012). From novel to familiar: tuning the brain for metaphors. Neuroimage 59, 3212-3221. doi: 10.1016/j.neuroimage.2011.11.079

Carlsson, I., Wendt, P. E., and Risberg, J. (2000). On the neurobiology of creativity. Differences in frontal activity between high and low creative subjects. Neuropsychologia 38, 873-885. doi: 10.1016/S0028-3932(99)00128-1

Catani, M., and Thiebaut de Schotten, M. (2012). Atlas of Human Brain Connections. New York, NY: Oxford University Press. doi: 10.1093/med/9780199541164.001.0001

Chakravarty, A. (2011). De novo development of artistic creativity in Alzheimer's disease. Ann. Indian Acad. Neurol. 14, 291-294. doi: 10.4103/0972-2327.91953

Changeux, J. (2005). "Creation, art and brain," in Neurobiology of Human Values, eds J.-P. Changeux, A. R. Damasio, W. Singer, and Y. Christen (Berlin; Heidelberg: Springer-Verlan), 1-11. doi: 10.1007/3-540-29803-7_1

Chatterjee, A., Bromberger, B., Smith, W. B. I. I., Sternschein, R., and Widick, P. (2011). Artistic production following brain damage: a study of three artists. Leonardo 44, 405-410. doi: 10.1162/LEON_a_00240

Chatterjee, A., Hamilton, R. H., and Amorapanth, P. X. (2006). Art produced by a patient with Parkinson's disease. Behav. Neurol. 17, 105-108. doi: $10.1155 / 2006 / 901832$

Chavez-Eakle, R. A., Graff-Guerrero, A., Garcia-Reyna, J. C., Vaugier, V., and Cruz-Fuentes, C. (2007). Cerebral blood flow associated with creative performance: a comparative study. Neuroimage 38, 519-528. doi: 10.1016/j.neuroimage.2007.07.059

Christoff, K., Keramatian, K., Gordon, A. M., Smith, R., and Madler, B. (2009). Prefrontal organization of cognitive control according to levels of abstraction. Brain Res. 1286, 94-105. doi: 10.1016/j.brainres.2009.05.096

Christoff, K., Prabhakaran, V., Dorfman, J., Zhao, Z., Kroger, J. K., Holyoak, K. J., et al. (2001). Rostrolateral prefrontal cortex involvement in relational integration during reasoning. Neuroimage 14, 1136-1149. doi: 10.1006/nimg.2001.0922

Chrysikou, E. G., and Thompson-Schill, S. L. (2011). Dissociable brain states linked to common and creative object use. Hum. Brain Mapp. 32, 665-675. doi: $10.1002 / \mathrm{hbm} .21056$

Chrysikou, E. G., Weber, M. J., and Thompson-Schill, S. L. (2013). A matched filter hypothesis for cognitive control. Neuropsychologia. doi: 10.1016/ j.neuropsychologia.2013.10.021. [Epub ahead of print].

Cole, M. W., Reynolds, J. R., Power, J. D., Repovs, G., Anticevic, A., and Braver, T. S. (2013). Multi-task connectivity reveals flexible hubs for adaptive task control. Nat. Neurosci. 16, 1348-1355. doi: 10.1038/nn.3470

Crutch, S. J., Isaacs, R., and Rossor, M. N. (2001). Some workmen can blame their tools: artistic change in an individual with Alzheimer's disease. Lancet 357, 2129-2133. doi: 10.1016/S0140-6736(00)05187-4

Cummings, J. L., and Zarit, J. M. (1987). Probable Alzheimer's disease in an artist. JAMA 258, 2731-2734. doi: 10.1001/jama.1987.03400190113039 de Manzano, O., and Ullen, F. (2012). Goal-independent mechanisms for free response generation: creative and pseudo-random performance share neural substrates. Neuroimage 59, 772-780. doi: 10.1016/j.neuroimage.2011. 07.016

de Souza, L. C., Volle, E., Bertoux, M., Czernecki, V., Funkiewiez, A., Allali, G., et al. (2010). Poor creativity in frontotemporal dementia: a window into the neural bases of the creative mind. Neuropsychologia 48, 3733-3742. doi: 10.1016/j.neuropsychologia.2010.09.010

Dietrich, A. (2004). The cognitive neuroscience of creativity. Psychon. Bull. Rev. 11, 1011-1026. doi: 10.3758/BF03196731

Dietrich, A., and Kanso, R. (2010). A review of EEG, ERP, and neuroimaging studies of creativity and insight. Psychol. Bull. 136, 822-848. doi: 10.1037/a0019749

Dijksterhuis, A., and Meurs, T. (2006). Where creativity resides: the generative power of unconscious thought. Conscious. Cogn. 15, 135-146. doi: 10.1016/j.concog.2005.04.007

Dorfman, L., Martindale, C., Gassimova, V., and Vartanian, O. (2008). Creativity and speed of information processing: a double dissociation involving elementary versus inhibitory cognitive tasks. Pers. Individ. Dif. 44, 1382-1390. doi: 10.1016/j.paid.2007.12.006

Drago, V., Crucian, G. P., Foster, P. S., Cheong, J., Finney, G. R., Pisani, F., et al. (2006a). Lewy body dementia and creativity: case report. Neuropsychologia 44, 3011-3015. doi: 10.1016/j.neuropsychologia.2006.05.030

Drago, V., Foster, P. S., Trifiletti, D., FitzGerald, D. B., Kluger, B. M., Crucian, G. P., et al. (2006b). What's inside the art? The influence of frontotemporal dementia in art production. Neurology 67, 1285-1287. doi: 10.1212/01.wnl.0000238439.77764.da

Dubois, B., Levy, R., Verin, M., Teixeira, C., Agid, Y., and Pillon, B. (1995). Experimental approach to prefrontal functions in humans. Ann. N.Y. Acad. Sci. 769, 41-60. doi: 10.1111/j.1749-6632.1995.tb38130.x

Edwards-Lee, T., Miller, B. L., Benson, D. F., Cummings, J. L., Russell, G. L., Boone, K., et al. (1997). The temporal variant of frontotemporal dementia. Brain $120(\mathrm{Pt}$ 6), 1027-1040. doi: 10.1093/brain/120.6.1027

Eickhoff, S. B., Bzdok, D., Laird, A. R., Kurth, F., and Fox, P. T. (2012). Activation likelihood estimation meta-analysis revisited. Neuroimage 59, 2349-2361. doi: 10.1016/j.neuroimage.2011.09.017

Ellamil, M., Dobson, C., Beeman, M., and Christoff, K. (2012). Evaluative and generative modes of thought during the creative process. Neuroimage 59, 1783-1794. doi: 10.1016/j.neuroimage.2011.08.008

Espinel, C. H. (1996). de Kooning's late colours and forms: dementia, creativity, and the healing power of art. Lancet 347, 1096-1098. doi: 10.1016/S01406736(96) $90285-8$

Fellows, L. K. (2013). "Decision-making: executive functions meet motivation," in Principles of Frontal Lobe Function, 2nd Edn., eds D. T. Stuss and R. T. Knight (New York, NY: Oxford University Press), 490-500.

Fink, A., Grabner, R. H., Benedek, M., Reishofer, G., Hauswirth, V., Fally, M., et al. (2009). The creative brain: investigation of brain activity during creative problem solving by means of EEG and FMRI. Hum. Brain Mapp. 30, 734-748. doi: 10.1002/hbm. 20538

Fink, A., Grabner, R. H., Gebauer, D., Reishofer, G., Koschutnig, K., and Ebner, F. (2010). Enhancing creativity by means of cognitive stimulation: evidence from an fMRI study. Neuroimage 52, 1687-1695. doi: 10.1016/j.neuroimage.2010.05.072

Finkelstein, Y., Vardi, J., and Hod, I. (1991). Impulsive artistic creativity as a presentation of transient cognitive alterations. Behav. Med. 17, 91-94. doi: 10.1080/08964289.1991.9935164

Finney, G. R., and Heilman, K. M. (2007). Artwork before and after onset of progressive nonfluent aphasia. Cogn. Behav. Neurol. 20, 7-10. doi: 10.1097/WNN.0b013e31802b6clf

Folstein, M. F., Folstein, S. E., and McHugh, P. R. (1975). "Mini-mental state." A practical method for grading the cognitive state of patients for the clinician. J. Psychiatr. Res. 12, 189-198. doi: 10.1016/0022-3956(75)90026-6

Fornazzari, L. R. (2005). Preserved painting creativity in an artist with Alzheimer's disease. Eur. J. Neurol. 12, 419-424. doi: 10.1111/j.1468-1331.2005.01128.x

Fuster, J. M., Bodner, M., and Kroger, J. K. (2000). Cross-modal and crosstemporal association in neurons of frontal cortex. Nature 405, 347-351. doi: $10.1038 / 35012613$

Gabora, L. (2010). Revenge of the "Neurds": characterizing creative thought in terms of the structure and dynamics of memory. Creat. Res. J. 22, 1-13. doi: 10.1080/10400410903579494 
Galarza, M., Isaac, C., Pellicer, O., Mayes, A., Broks, P., Montaldi, D., et al. (2014). Jazz, guitar, and neurosurgery: the Pat Martino case report. World Neurosurg. 81, 651.el-e7. doi: 10.1016/j.wneu.2013.09.042

Gansler, D. A., Moore, D. W., Susmaras, T. M., Jerram, M. W., Sousa, J., and Heilman, K. M. (2011). Cortical morphology of visual creativity. Neuropsychologia 49, 2527-2532. doi: 10.1016/j.neuropsychologia.2011.05.001

Geake, J. G., and Hansen, P. C. (2005). Neural correlates of intelligence as revealed by fMRI of fluid analogies. Neuroimage 26, 555-564. doi: 10.1016/j.neuroimage.2005.01.035

Gilbert, S. J., Dumontheil, I., Simons, J. S., Frith, C. D., and Burgess, P. W. (2007). Comment on "Wandering minds: the default network and stimulusindependent thought." Science 317, 43; author reply 43. doi: 10.1126/science. 1140801

Gilbert, S. J., Gonen-Yaacovi, G., Benoit, R. G., Volle, E., and Burgess, P. W. (2010). Distinct functional connectivity associated with lateral versus medial rostral prefrontal cortex: a meta-analysis. Neuroimage 53, 1359-1367. doi: 10.1016/j.neuroimage.2010.07.032

Godefroy, O. (2003). Frontal syndrome and disorders of executive functions. J. Neurol. 250, 1-6. doi: 10.1007/s00415-003-0918-2

Goel, V., and Vartanian, O. (2005). Dissociating the roles of right ventral lateral and dorsal lateral prefrontal cortex in generation and maintenance of hypotheses in set-shift problems. Cereb. Cortex 15, 1170-1177. doi: 10.1093/cercor/bhh217

Goldman-Rakic, P. S. (1995). Architecture of the prefrontal cortex and the central executive. Ann. N.Y. Acad. Sci. 769, 71-83. doi: 10.1111/j.17496632.1995.tb38132.x

Gonen-Yaacovi, G., de Souza, L. C., Levy, R., Urbanski, M., Josse, G., and Volle, E. (2013). Rostral and caudal prefrontal contribution to creativity: a meta-analysis of functional imaging data. Front. Hum. Neurosci. 7:465. doi: 10.3389/fnhum.2013.00465

Green, A. E., Kraemer, D. J. M., Fugelsang, J. A., Gray, J. R., and Dunbar, K. N. (2012). Neural correlates of creativity in analogical reasoning. J. Exp. Psychol. Learn. Mem. Cogn. 38, 264-272. doi: 10.1037/a0025764

Gretton, C., and ffytche, D. H. (2014). Art and the brain: a view from dementia. Int. J. Geriatr. Psychiatry 29, 111-126. doi: 10.1002/gps.3975

Howard-Jones, P. A., Blakemore, S. J., Samuel, E. A., Summers, I. R., and Claxton, G. (2005). Semantic divergence and creative story generation: an fMRI investigation. Brain Res. Cogn. Brain Res. 25, 240-250. doi: 10.1016/j.cogbrainres.2005.05.013

Huang, P., Qiu, L., Shen, L., Zhang, Y., Song, Z., Qi, Z., et al. (2013). Evidence for a left-over-right inhibitory mechanism during figural creative thinking in healthy nonartists. Hum. Brain Mapp. 34, 2724-2732. doi: 10.1002/hbm.22093

Jung, R. E. (2013). The structure of creative cognition in the human brain. Front. Hum. Neurosci. 7: 330. doi: 10.3389/fnhum.2013.00330

Jung, R. E., Grazioplene, R., Caprihan, A., Chavez, R. S., and Haier, R. J. (2010a). White matter integrity, creativity, and psychopathology: disentangling constructs with diffusion tensor imaging. PLOS ONE 5:e9818. doi: 10.1371/journal.pone.0009818

Jung, R. E., Segall, J. M., Jeremy Bockholt, H., Flores, R. A., Smith, S. M., Chavez, R. S., et al. (2010b). Neuroanatomy of creativity. Hum. Brain Mapp. 31, 398-409. doi: $10.1002 / \mathrm{hbm} .20874$

Jung-Beeman, M., Bowden, E. M., Haberman, J., Frymiare, J. L., Arambel-Liu, S., Greenblatt, R., et al. (2004). Neural activity when people solve verbal problems with insight. PLoS Biol. 2:E97. doi: 10.1371/journal.pbio.0020097

Kapur, N. (1996). Paradoxical functional facilitation in brain-behaviour research. A critical review. Brain 119(Pt 5), 1775-90. doi: 10.1093/brain/119.5.1775

Kim, K. H. (2006). Can we trust creativity tests? A review of the torrance tests of creative thinking (TTCT). Creat. Res. J. 18, 3-14. doi: 10.1207/s15326934crj1801_2

Kleiner-Fisman, G., Black, S. E., and Lang, A. E. (2003). Neurodegenerative disease and the evolution of art: the effects of presumed corticobasal degeneration in a professional artist. Mov. Disord. 18, 294-302. doi: 10.1002/mds.10360

Koechlin, E., and Hyafil, A. (2007). Anterior prefrontal function and the limits of human decision-making. Science 318, 594-598. doi: 10.1126/science.1142995

Koechlin, E., Ody, C., and Kouneiher, F. (2003). The architecture of cognitive control in the human prefrontal cortex. Science 302, 1181-1185. doi: 10.1126/science. 1088545

Kounios, J., Frymiare, J. L., Bowden, E. M., Fleck, J. I., Subramaniam, K., Parrish, T. B., et al. (2006). The prepared mind: neural activity prior to problem presentation predicts subsequent solution by sudden insight. Psychol. Sci. 17, 882-890. doi: 10.1111/j.1467-9280.2006.01798.x
Kowatari, Y., Lee, S. H., Yamamura, H., Nagamori, Y., Levy, P., Yamane, S., et al. (2009). Neural networks involved in artistic creativity. Hum. Brain Mapp. 30, 1678-1690. doi: 10.1002/hbm.20633

Kozbelt, A. (2011). "Theories of creativity," in Encyclopedia of Creativity, 2nd Edn., eds M. A. Runco and S. R. Pritzker (San Diego, CA: Academic Press), 473-479. doi: 10.1016/B978-0-12-375038-9.00223-5

Kröger, S., Rutter, B., Stark, R., Windmann, S., Hermann, C., and Abraham, A. (2012). Using a shoe as a plant pot: neural correlates of passive conceptual expansion. Brain Res. 1430, 52-61. doi: 10.1016/j.brainres.2011.10.031

Levy, B. J., and Wagner, A. D. (2011). Cognitive control and right ventrolateral prefrontal cortex: reflexive reorienting, motor inhibition, and action updating. Ann. N.Y. Acad. Sci. 1224, 40-62. doi: 10.1111/j.1749-6632.2011.05958.x

Levy, R., and Volle, E. (2009). [The prefrontal cortex: composer and conductor of voluntary behaviors]. Rev. Neurol. (Paris). 165 Spec No 3, F159-F177.

Lhommée, E., Batir, A., Quesada, J. L., Ardouin, C., Fraix, V., Seigneuret, E., et al. (2014). Dopamine and the biology of creativity: lessons from Parkinson's disease. Front. Neurol. 5:55. doi: 10.3389/fneur.2014.00055

Limb, C. J., and Braun, A. R. (2008). Neural substrates of spontaneous musical performance: an fMRI study of jazz improvisation. PLoS ONE 3:e1679. doi: 10.1371/journal.pone.0001679

Liu, A., Werner, K., Roy, S., Trojanowski, J. Q., Morgan-Kane, U., Miller, B. L., et al. (2009). A case study of an emerging visual artist with frontotemporal lobar degeneration and amyotrophic lateral sclerosis. Neurocase 15, 235-247. doi: 10.1080/13554790802633213

Luo, J., Niki, K., and Phillips, S. (2004). Neural correlates of the 'Aha! reaction'. Neuroreport 15, 2013-2017. doi: 10.1097/00001756-200409150-00004

Lythgoe, M. F., Pollak, T. A., Kalmus, M., de Haan, M., and Chong, W. K. (2005). Obsessive, prolific artistic output following subarachnoid hemorrhage. Neurology 64, 397-398. doi: 10.1212/01.WNL.0000150526.09499.3E

Malloy-Diniz, L. F., Lasmar, V. A., Gazinelli Lde, S., Fuentes, D., and Salgado, J. V. (2007). The Rey Auditory-Verbal Learning Test: applicability for the Brazilian elderly population. Rev. Bras. Psiquiatr. 29, 324-329. doi: 10.1590/S151644462006005000053

Martin, R. C., and Cheng, Y. (2006). Selection demands versus association strength in the verb generation task. Psychon. Bull. Rev. 13, 396-401. doi: 10.3758/BF03193859

Mashal, N., Faust, M., Hendler, T., and Jung-Beeman, M. (2007). An fMRI investigation of the neural correlates underlying the processing of novel metaphoric expressions. Brain Lang. 100, 115-126. doi: 10.1016/j.bandl.2005.10.005

Mason, M. F., Norton, M. I., Van Horn, J. D., Wegner, D. M., Grafton, S. T., and Macrae, C. N. (2007). Wandering minds: the default network and stimulusindependent thought. Science 315, 393-395. doi: 10.1126/science.1131295

Maurer, K., and Prvulovic, D. (2004). Paintings of an artist with Alzheimer's disease: visuoconstructural deficits during dementia. J. Neural. Transm. 111, 235-245. doi: 10.1007/s00702-003-0046-2

Mednick, M. T., Mednick, S. A., and Jung, C. C. (1964). Continual association as a function of level of creativity and type of verbal stimulus. J. Abnorm. Psychol. 69, 511-515. doi: 10.1037/h0041086

Mednick, S. A. (1962). The associative basis of the creative process. Psychol. Rev. 69, 220-232. doi: 10.1037/h0048850

Mell, J. C., Howard, S. M., and Miller, B. L. (2003). Art and the brain: the influence of frontotemporal dementia on an accomplished artist. Neurology 60 , 1707-1710. doi: 10.1212/01.WNL.0000064164.02891.12

Mendez, M. F. (2004). Dementia as a window to the neurology of art. Med. Hypotheses 63, 1-7. doi: 10.1016/j.mehy.2004.03.002

Mendez, M. F., and Perryman, K. M. (2003). Disrupted facial empathy in drawings from artists with frontotemporal dementia. Neurocase 9, 44-50. doi: 10.1076/neur.9.1.44.14375

Mesulam, M. M. (1998). From sensation to cognition. Brain 121(Pt 6), 1013-1052. doi: 10.1093/brain/121.6.1013

Midorikawa, A., Fukutake, T., and Kawamura, M. (2008). Dementia and painting in patients from different cultural backgrounds. Eur. Neurol. 60, 224-229. doi: $10.1159 / 000151697$

Miller, B. L., Boone, K., Cummings, J. L., Read, S. L., and Mishkin, F. (2000). Functional correlates of musical and visual ability in frontotemporal dementia. Br. J. Psychiatry 176, 458-463. doi: 10.1192/bjp.176.5.458

Miller, B. L., Cummings, J., Mishkin, F., Boone, K., Prince, F., Ponton, M., et al. (1998). Emergence of artistic talent in frontotemporal dementia. Neurology 51, 978-982. doi: 10.1212/WNL.51.4.978 
Miller, B. L., and Hou, C. E. (2004). Portraits of artists: emergence of visual creativity in dementia. Arch. Neurol. 61, 842-844. doi: 10.1001/archneur.61.6.842

Miller, B. L., Ponton, M., Benson, D. F., Cummings, J. L., and Mena, I. (1996). Enhanced artistic creativity with temporal lobe degeneration. Lancet 348, 1744-1745. doi: 10.1016/S0140-6736(05)65881-3

Miller, E. K., and Cohen, J. D. (2001). An integrative theory of prefrontal cortex function. Annu. Rev. Neurosci. 24, 167-202. doi: 10.1146/annurev.neuro.24. 1.167

Nee, D. E., Jahn, A., and Brown, J. W. (2013). Prefrontal cortex organization: dissociating effects of temporal abstraction, relational abstraction, and integration with fMRI. Cereb. Cortex. doi: 10.1093/cercor/bht091. [Epub ahead of print].

Palmiero, M., Di Giacomo, D., and Passafiume, D. (2012). Creativity and dementia: a review. Cogn. Process. 13, 193-209. doi: 10.1007/s10339-012-0439-y

Pfeffer, R. I., Kurosaki, T. T., Harrah, C. H. Jr., Chance, J. M., and Filos, S. (1982). Measurement of functional activities in older adults in the community. J. Gerontol. 37, 323-329. doi: 10.1093/geronj/37.3.323

Picton, T. W., Stuss, D. T., Alexander, M. P., Shallice, T., Binns, M. A., and Gillingham, S. (2007). Effects of focal frontal lesions on response inhibition. Cereb. Cortex 17, 826-838. doi: 10.1093/cercor/bhk031

Plucker, J. A., and Makel, M. C. (2010). "Assessment of creativity," in The Cambridge Handbook of Creativity, eds J. C. Kaufman and R. J. Sternberg (Cambridge: Cambridge University Press), 48-73. doi: 10.1017/CBO9780511763205.005

Porto, C. S., Fichman, H. C., Caramelli, P., Bahia, V. S., and Nitrini, R. (2003). Brazilian version of the Mattis dementia rating scale: diagnosis of mild dementia in Alzheimer's disease. Arq. Neuropsiquiatr. 61, 339-345. doi: 10.1590/S0004282X2003000300004

Qiu, J., Li, H., Jou, J., Liu, J., Luo, Y., Feng, T., et al. (2010). Neural correlates of the "Aha" experiences: evidence from an fMRI study of insight problem solving. Cortex 46, 397-403. doi: 10.1016/j.cortex.2009.06.006

Quental, N. B., Brucki, S. M., and Bueno, O. F. (2013). Visuospatial function in early Alzheimer's disease-the use of the Visual Object and Space Perception (VOSP) battery. PLOS ONE 8:e68398. doi: 10.1371/journal.pone.0068398

Ramnani, N., and Owen, A. M. (2004). Anterior prefrontal cortex: insights into function from anatomy and neuroimaging. Nat. Rev. Neurosci. 5, 184-194. doi: 10.1038/nrn1343

Rankin, K. P., Liu, A. A., Howard, S., Slama, H., Hou, C. E., Shuster, K., et al. (2007). A case-controlled study of altered visual art production in Alzheimer's and FTLD. Cogn. Behav. Neurol. 20, 48-61. doi: 10.1097/WNN.0b013e31803141dd

Rascovsky, K., Hodges, J. R., Knopman, D., Mendez, M. F., Kramer, J. H., Neuhaus, J., et al. (2011). Sensitivity of revised diagnostic criteria for the behavioural variant of frontotemporal dementia. Brain 134, 2456-2477. doi: 10.1093/brain/awr179

Reverberi, C., Toraldo, A., D’Agostini, S., and Skrap, M. (2005). Better without (lateral) frontal cortex? Insight problems solved by frontal patients. Brain 128, 2882-2890. doi: 10.1093/brain/awh577

Reynolds, J. R., McDermott, K. B., and Braver, T. S. (2006). A direct comparison of anterior prefrontal cortex involvement in episodic retrieval and integration. Cereb. Cortex 16, 519-528. doi: 10.1093/cercor/bhi131

Ritter, S. M., and Dijksterhuis, A. (2014). Creativity-the unconscious foundations of the incubation period. Front. Hum. Neurosci. 8:215. doi: 10.3389/fnhum.2014.00215

Runco, M. A. (2004). Creativity. Annu. Rev. Psychol. 55, 657-687. doi: 10.1146/annurev.psych.55.090902.141502

Runco, M. A., and Acar, S. (2012). Divergent thinking as an indicator of creative potential. Creat. Res. J. 24, 66-75. doi: 10.1080/10400419.2012.652929

Rutter, B., Kröger, S., Stark, R., Schweckendiek, J., Windmann, S., Hermann, C., et al. (2012). Can clouds dance? Neural correlates of passive conceptual expansion using a metaphor processing task: implications for creative cognition. Brain Cogn. 78, 114-122. doi: 10.1016/j.bandc.2011.11.002

Sahlas, D. J. (2003). Dementia with Lewy bodies and the neurobehavioral decline of Mervyn Peake. Arch. Neurol. 60, 889-892. doi: 10.1001/archneur.60.6.889

Schoenbaum, G., Roesch, M. R., Stalnaker, T. A., and Takahashi, Y. K. (2009). A new perspective on the role of the orbitofrontal cortex in adaptive behaviour. Nat. Rev. Neurosci. 10, 885-892. doi: 10.1038/nrn2753

Schott, G. D. (2012). Pictures as a neurological tool: lessons from enhanced and emergent artistry in brain disease. Brain 135, 1947-1963. doi: 10.1093/brain/awr314

Schrag, A., and Trimble, M. (2001). Poetic talent unmasked by treatment of Parkinson's disease. Mov. Disord. 16, 1175-1176. doi: 10.1002/mds.1239
Seeley, W. W., Matthews, B. R., Crawford, R. K., Gorno-Tempini, M. L., Foti, D., Mackenzie, I. R., et al. (2008). Unravelling Boléro: progressive aphasia, transmodal creativity and the right posterior neocortex. Brain 131, 39-49. doi: 10.1093/brain/awm270

Seger, C. A., Desmond, J. E., Glover, G. H., and Gabrieli, J. D. (2000). Functional magnetic resonance imaging evidence for right-hemisphere involvement in processing unusual semantic relationships. Neuropsychology 14, 361-369. doi: 10.1037/0894-4105.14.3.361

Serrano, C., Allegri, R. F., Martelli, M., Taragano, F., and Rinalli, P. (2005). [Visual art, creativity and dementia]. Vertex 16, 418-429.

Shah, C., Erhard, K., Ortheil, H.-J., Kaza, E., Kessler, C., and Lotze, M. (2013). Neural correlates of creative writing: an fMRI Study. Hum. Brain Mapp. 34, 1088-1101. doi: 10.1002/hbm.21493

Shallice, T., and Burgess, P. (1996). The domain of supervisory processes and temporal organization of behaviour. Philos. Trans. R. Soc. Lond. B Biol. Sci. 351, 1405-1411; discussion 1411-1412. doi: 10.1098/rstb.1996.0124

Shamay-Tsoory, S. G., Adler, N., Aharon-Peretz, J., Perry, D., and Mayseless, N. (2011). The origins of originality: the neural bases of creative thinking and originality. Neuropsychologia 49, 178-185. doi: 10.1016/j.neuropsychologia.2010.11.020

Siebörger, F. T., Ferstl, E. C., and von Cramon, D. Y. (2007). Making sense of nonsense: an fMRI study of task induced inference processes during discourse comprehension. Brain Res. 1166, 77-91. doi: 10.1016/j.brainres.2007. 05.079

Simonton, D. K. (2010). Creative thought as blind-variation and selectiveretention: combinatorial models of exceptional creativity. Phys. Life Rev. 7, 190-194. doi: 10.1016/j.plrev.2010.05.004

Spreng, R. N., Stevens, W. D., Chamberlain, J. P., Gilmore, A. W., and Schacter, D. L. (2010). Default network activity, coupled with the frontoparietal control network, supports goal-directed cognition. Neuroimage 53, 303-317. doi: 10.1016/j.neuroimage.2010.06.016

Sternberg, R. J. (2006). The nature of creativity. Creat. Res. J. 18, 87. doi: 10.1207/s15326934crj1801_10

Sternberg, R. J. L., and Lubart, T. (1999). "The concept of creativity: prospects and paradigms," in Handbook of Creativity, ed R. J. Sternberg (Cambridge: Cambridge University Press), 3-15.

Strenziok, M., Greenwood, P. M., Santa Cruz, S. A., Thompson, J. C., and Parasuraman, R. (2013). Differential contributions of dorso-ventral and rostrocaudal prefrontal white matter tracts to cognitive control in healthy older adults. PLoS ONE 8:e81410. doi: 10.1371/journal.pone.0081410

Takahata, K., Saito, F., Muramatsu, T., Yamada, M., Shirahase, J., Tabuchi, H., et al. (2014). Emergence of realism: enhanced visual artistry and high accuracy of visual numerosity representation after left prefrontal damage. Neuropsychologia 57, 38-49. doi: 10.1016/j.neuropsychologia.2014.02.022

Takeuchi, H., Taki, Y., Hashizume, H., Sassa, Y., Nagase, T., Nouchi, R., et al. (2012). The association between resting functional connectivity and creativity. Cereb. Cortex 22, 2921-2929. doi: 10.1093/cercor/bhr371

Takeuchi, H., Taki, Y., Sassa, Y., Hashizume, H., Sekiguchi, A., Fukushima, A., et al. (2010a). Regional gray matter volume of dopaminergic system associate with creativity: evidence from voxel-based morphometry. Neuroimage 51, 578-585. doi: 10.1016/j.neuroimage.2010.02.078

Takeuchi, H., Taki, Y., Sassa, Y., Hashizume, H., Sekiguchi, A., Fukushima, A., et al. (2010b). White matter structures associated with creativity: evidence from diffusion tensor imaging. Neuroimage 51, 11-18. doi: 10.1016/j.neuroimage.2010.02.035

Thomas-Anterion, C. (2009). Emergence of artist talent and neurology: three cases. Rev. Neuropsychol. 1, 221-228. doi: 10.1684/nrp.2009.0029

Thomas-Anterion, C., Creac'h, C., Dionet, E., Borg, C., Extier, C., Faillenot, I., et al. (2010). De novo artistic activity following insular-SII ischemia. Pain 150, 121-127. doi: 10.1016/j.pain.2010.04.010

Thomas-Anterion, C., Honore-Masson, S., Dirson, S., and Laurent, B. (2002). Lonely cowboy's thoughts. Neurology 59, 1812-1813. doi: 10.1212/01.WNL.0000035637.48621.68

Thompson-Schill, S. L., and Botvinick, M. M. (2006). Resolving conflict: a response to Martin and Cheng (2006). Psychon. Bull. Rev. 13, 402-408; discussion: 09-11.

Tian, F., Tu, S., Qiu, J., Lv, J. Y., Wei, D. T., Su, Y. H., et al. (2011). Neural correlates of mental preparation for successful insight problem solving. Behav. Brain Res. 216, 626-630. doi: 10.1016/j.bbr.2010.09.005 
Torrance, E. P. (2004). Un résumé historique du développement des tests de pensée créative de Torrance. Rev. Eur. Psychol. Appl. 54, 57-63. doi: 10.1016/j.erap.2004.01.003

van Buren, B., Bromberger, B., Potts, D., Miller, B., and Chatterjee, A. (2013). Changes in painting styles of two artists with Alzheimer's disease. Psychol. Aesthet. Creat. Arts 7, 89-94. doi: 10.1037/a0029332

Vartanian, O., and Goel, V. (2005). Task constraints modulate activation in right ventral lateral prefrontal cortex. Neuroimage 27, 927-933. doi: 10.1016/j.neuroimage.2005.05.016

Vartanian, O., Martindale, C., and Kwiatkowski, J. (2007). Creative potential, attention, and speed of information processing. Pers. Individ. Dif. 43, 1470-1480. doi: 10.1016/j.paid.2007.04.027

Volle, E., de Lacy Costello, A., Coates, L. M., McGuire, C., Towgood, K., Gilbert, S., et al. (2012). Dissociation between verbal response initiation and suppression after prefrontal lesions. Cereb. Cortex 22, 2428-2440. doi: 10.1093/cercor/bhr322

Volle, E., Gilbert, S. J., Benoit, R. G., and Burgess, P. W. (2010). Specialization of the rostral prefrontal cortex for distinct analogy processes. Cereb. Cortex 20, 2647-2659. doi: 10.1093/cercor/bhq012

Volle, E., Levy, R., and Burgess, P. W. (2013). "A new era for lesion-behavior mapping of prefrontal functions," in Principles of Frontal Lobe Function, 2nd edn., eds D. T. Stuss and R. T. Knight (New York, NY: Oxford University Press), 500-523.

Ward, T. B., and Kolomyts, Y. (2010). "Cognition and creativity," in The Cambridge Handbook of Creativity, eds J. C. Kaufman and R. J. Sternberg (Cambridge: Cambridge University Press), 93-112. doi: 10.1017/CBO9780511763205.008

Warrington, E. K., and James, M. (1991). Visual Object and Space Perception Battery. Bury St Edmunds: Thames Valley Test Company

Wise, R. J., and Braga, R. M. (2014). Default mode network: the seat of literary creativity? Trends Cogn. Sci. 18, 116-117. doi: 10.1016/j.tics.2013.11.001
Wu, T. Q., Miller, Z. A., Adhimoolam, B., Zackey, D. D., Khan, B. K., Ketelle, R., et al. (2013). Verbal creativity in semantic variant primary progressive aphasia. Neurocase doi: 10.1080/13554794.2013.860179. [Epub ahead of print].

Yaniv, I., and Meyer, D. E. (1987). Activation and metacognition of inaccessible stored information: potential bases for incubation effects in problem solving. J. Exp. Psychol. Learn. Mem. Cogn. 13, 187-205. doi: 10.1037/02787393.13.2.187

Yeterian, E. H., Pandya, D. N., Tomaiuolo, F., and Petrides, M. (2012). The cortical connectivity of the prefrontal cortex in the monkey brain. Cortex 48, 58-81. doi: 10.1016/j.cortex.2011.03.004

Zhong, C. B., Dijksterhuis, A., and Galinsky, A. D. (2008). The merits of unconscious thought in creativity. Psychol. Sci. 19, 912-918. doi: 10.1111/j.14679280.2008.02176.x

Conflict of Interest Statement: The authors declare that the research was conducted in the absence of any commercial or financial relationships that could be construed as a potential conflict of interest.

Received: 05 June 2014; accepted: 28 June 2014; published online: 23 July 2014.

Citation: de Souza LC, Guimarães HC, Teixeira AL, Caramelli P, Levy R, Dubois B and Volle E (2014) Frontal lobe neurology and the creative mind. Front. Psychol. 5:761. doi: 10.3389/fpsyg.2014.00761

This article was submitted to Psychopathology, a section of the journal Frontiers in Psychology.

Copyright (c) 2014 de Souza, Guimarães, Teixeira, Caramelli, Levy, Dubois and Volle. This is an open-access article distributed under the terms of the Creative Commons Attribution License (CC BY). The use, distribution or reproduction in other forums is permitted, provided the original author(s) or licensor are credited and that the original publication in this journal is cited, in accordance with accepted academic practice. No use, distribution or reproduction is permitted which does not comply with these terms. 